\title{
A KINETIC MODEL ON PORTFOLIO IN FINANCE*
}

\author{
JIAHANG $\mathrm{CHE}^{\dagger}$
}

\begin{abstract}
In this paper a multi-dimensional simplified kinetic model (following the ideas of onedimensional model by Cordier, Pareschi, Toscani [S. Cordier, L. Pareschi, and G. Toscani, J. Stat. Phys., 120, 253-277, 2005]) which uses Mossin's expression for portfolio [J. Mossin, Econometrica: Journal of the Econometric Society, 768-783, 1966] is established to describe the time evolution of the portfolio distribution for several risky assets in the market. The existence and uniqueness of $L^{1}$-solutions of the model and the $L^{1}$-weak compactness of the time-scaled solutions are proved. Furthermore, the limit of the time-scaled solutions is proved to satisfy a weak form of the multidimensional Fokker-Planck equation under some assumptions on the parameters in the trading rule.
\end{abstract}

Key words. Econophysics, portfolio, Boltzmann equation.

AMS subject classifications. 91B60, 35B40.

\section{Introduction}

Econophysics is an interdisciplinary field involving economics and physics, which was developed two decades ago. It has received a lot of attention recently, in particular on its applications to the financial markets. Several models were investigated by means of the basic ideas and tools from statistical physics, which offer a new way to the research of complicated economic systems (see, e.g., $[2,4,6,7,10]$ ). In these models, the agents and their transactions in the market can be identified with molecules and molecular interactions in physics respectively. The main reason is that the economic systems, which consist of a large number of agents, are analogue to the physical systems composed of many particles.

Since the inverse power law was demonstrated by V.Pareto [21] more than one century ago, there have been a lot of papers on the wealth distribution verifying his conclusion. The model proposed by Drăgulescu and Yakovenko [10], in which money is considered to be a conserved quantity, showed that the stationary distribution admits an exponential Boltzmann-Gibbs function. Another model was established by Chakraborti and Chakrabarti [5], where the saving propensity ensures that all the individuals don't spend up their money and plays an important role in the trading rule. Recently, Cordier, Pareschi and Toscani [9] derived a one-dimensional Boltzmann-like equation for a simple market economy with a somewhat different trading rule in which the random variable was introduced as an ingredient to characterize the rate of market returns. They also proved that the asymptotic limit of the time-scaled distribution obeys a one-dimensional Fokker-Planck equation. Later on, based on the Levy-LevySolomon model, a detailed model characterizing the portfolio ${ }^{1}$ between stock and bond was derived by Cordier, Pareschi, Piatecki [8]. Other models can also be found for instance in $[12,13,14,20]$. The readers can get more information on the research of econophysics from the review paper [25] in 2009 given by Yakovenko and Rosser.

Generally speaking, individuals or firms combine alternative investments into proper portfolios in order to reduce the total risk. Therefore, it is necessary to establish a multi-dimensional kinetic model to characterize the time evolution of the

\footnotetext{
${ }^{*}$ Received: August 4, 2010; accepted (in revised version): March 21, 2011. Communicated by Lorenzo Pareschi.

${ }^{\dagger}$ Department of Mathematical Sciences, Tsinghua University, Beijing, 100084, People's Republic of China (chejh05@mails.tsinghua.edu.cn).

${ }^{1}$ Portfolio: a set of various assets held by each individual or firm in order to diversify risk.
} 
portfolio distribution. Following the ideas in [9] and using the vector-expression of portfolio as in [18], we will establish a multi-dimensional model which might be helpful to describe the time evolution of the portfolio distribution in the financial market. Similar to the one-dimensional case, the random variable, whose variance measures the risk of transactions, is an essential factor in the trading rule of our model. Also, the characteristic functions are introduced in the Boltzmann-type equation of our model. These functions are used to guarantee that the debt is not allowed in the transaction, which is different from the molecular interactions in physics because the molecular interactions are unconditional in physics. Additionally, in the discussion of our multi-dimensional model, the trade is limited within the same kind of investment. In stock exchange, for example, one sells stocks of a company while another buys the stocks of the same company. This restriction is reasonable in view of this example, since the exchange among various kinds of stocks happens rarely.

It is very helpful for investors to know as thoroughly as possible the large time tendency of the investment in the financial market. For this purpose, one needs to study large time behavior of the portfolio distribution. Matthes and Toscani [17] studied a type of kinetic model which conserves the first moment; they derived a criterion which guarantees the existence of nontrivial stationary state. Our kinetic model has less symmetric structure and only conserves the zeroth moment. Therefore, it is difficult to obtain the nontrivial stationary state directly even in the sense of weak convergence, which is quite different from the classical Boltzmann equation and the case in [17]. As mentioned above, a feasible way to deal with the convergence problem has been given by $[8,9]$ for the one-dimensional model. We show that this also works for the multi-dimensional model. However it should be noted that here the time evolution of distribution in the multi-dimensional model is not simply a sum of those in one-dimensional models but depends on the relations between every two risky assets. It is well known that the covariance, which is often used to describe the relations between two risky assets in the economy, is usually not zero because risky asset prices are inevitably influenced by certain common factors. The appearance of correlation coefficients in multi-dimensional Fokker-Planck equation that we finally obtain shows the reason why we study the multi-dimensional model.

The rest of the paper is organized as follows: in Section 2 we first set up our multi-dimensional model by applying kinetic theory, and then prove the existence and uniqueness of mild solutions, and some related properties. Despite the fact that it can be more technical and involved, the existence and uniqueness should not be as big a problem for the present model as it is for the one-dimensional case in [9]. Here we will only present the main steps of the proof and omit some details. In Section 3 we prove some estimates which are crucial to the proof of the main theorem. In Section 4 we prove our main theorem.

\section{A multi-dimensional kinetic model for portfolio and main properties}

Using the same expression as Mossin in [18], we denote by $\mathbf{w}=\left(w_{1}, \cdots, w_{m}\right)^{T}$ and $\mathbf{w}_{*}=\left(w_{1 *}, \cdots, w_{m *}\right)^{T}$ the $m$-asset ( or $m$-stock ) portfolios of two arbitrary individuals. We assume that the $i$ th component of each portfolio represents the holdings of the portfolio in asset $i$, which is analogous to the $i$ th component of the velocity in physics. By virtue of this, the transaction is analogous to the collision.

Let $\boldsymbol{\eta}=\left(\eta_{1}, \cdots, \eta_{m}\right)^{T}, \boldsymbol{\eta}_{*}=\left(\eta_{1 *}, \cdots, \eta_{m *}\right)^{T}$ be two random vectors representing the rates of market returns. Their variances measure the risk (such as price fluctuation) according to Markowitz theory. Denote $\mathbf{x}=\left(x_{1}, \cdots, x_{m}\right)^{T}, \mathbf{x}_{*}=\left(x_{1 *}, \cdots, x_{m *}\right)^{T}$ as the 
outcomes of $\boldsymbol{\eta}$ and $\boldsymbol{\eta}_{*}$, respectively, i.e.

$$
\begin{gathered}
\mathbf{x}=\mathbf{x}(\boldsymbol{\eta})=\left(x_{1}(\boldsymbol{\eta}), \cdots, x_{m}(\boldsymbol{\eta})\right)^{T} \\
\mathbf{x}_{*}=\mathbf{x}_{*}\left(\boldsymbol{\eta}_{*}\right)=\left(x_{1 *}\left(\boldsymbol{\eta}_{*}\right), \cdots, x_{m *}\left(\boldsymbol{\eta}_{*}\right)\right)^{T} .
\end{gathered}
$$

Let us denote by $\mathbf{w}, \mathbf{w}_{*}$ the portfolios of two arbitrary individuals before the trade, and by $\mathbf{w}^{\prime}, \mathbf{w}_{*}^{\prime}$ the generated portfolios after the trade. Following the ideas in [9], we shall have the interaction rule

$$
\begin{aligned}
w_{i}^{\prime} & =\left(1-r_{i}\right) w_{i}+r_{i} w_{i *}+x_{i} w_{i}, \\
w_{i *}^{\prime} & =\left(1-r_{i}\right) w_{i *}+r_{i} w_{i}+x_{i *} w_{i *}, \quad 1 \leq i \leq m,
\end{aligned}
$$

where $0<r_{i}<1$. To see the dependence of $\mathbf{w}^{\prime}, \mathbf{w}_{*}^{\prime}$ on $\mathbf{w}, \mathbf{w}_{*}, \mathbf{r}=\left(r_{1}, \cdots, r_{m}\right)^{T}$ clearly, we rewrite (2.1)-(2.2) as

$$
\mathbf{w}^{\prime}=D(\mathbf{x}, \mathbf{r}) \mathbf{w}+D(\mathbf{r}) \mathbf{w}_{*}, \quad \mathbf{w}_{*}^{\prime}=D\left(\mathbf{x}_{*}, \mathbf{r}\right) \mathbf{w}_{*}+D(\mathbf{r}) \mathbf{w},
$$

with $D(\mathbf{x}, \mathbf{r}), D(\mathbf{r})$ being

$$
D(\mathbf{x}, \mathbf{r})=\operatorname{diag}\left\{1-r_{1}+x_{1}, \cdots, 1-r_{m}+x_{m}\right\}, \quad D(\mathbf{r})=\operatorname{diag}\left\{r_{1}, \cdots, r_{m}\right\} .
$$

Throughout this paper, we assume:

- $\boldsymbol{\eta}=\left(\eta_{1}, \cdots, \eta_{m}\right)^{T}$ and $\boldsymbol{\eta}_{*}=\left(\eta_{1 *}, \cdots, \eta_{m *}\right)^{T}$ are two random variable vectors with the same distribution. Denote the joint density function by $\rho:=\rho_{\boldsymbol{\eta}}$ for simplicity.

- $\rho(\mathbf{x})$ is obtained from a given random vector $\boldsymbol{\zeta}=\left(\zeta_{1}, \cdots, \zeta_{m}\right)^{T}$, i.e.

$$
\rho\left(x_{1}, \cdots, x_{m}\right)=\frac{1}{\prod_{i=1}^{m} \sigma_{i}} \rho_{0}\left(\frac{x_{1}}{\sigma_{1}}, \cdots, \frac{x_{m}}{\sigma_{m}}\right),
$$

where $\rho_{0}(\mathbf{x})$ is the joint density function of $\boldsymbol{\zeta}$ and satisfies

$$
\rho_{0}(\mathbf{x})=\rho_{0}(-\mathbf{x}), \quad \int_{\mathbb{R}^{m}} x_{i}^{2} \rho_{0}(\mathbf{x}) \mathrm{d} \mathbf{x}=1 .
$$

Thus it is easily seen that

$$
\int_{\mathbb{R}^{m}} x_{i} \rho(\mathbf{x}) \mathrm{d} \mathbf{x}=0, \quad \int_{\mathbb{R}^{m}} x_{i}^{2} \rho(\mathbf{x}) \mathrm{d} \mathbf{x}=\sigma_{i}^{2} .
$$

Let $f(\mathbf{w}, t)$ be the distribution of agents with the portfolio $\mathbf{w}=\left(w_{1}, \cdots, w_{m}\right)^{T}$ at the time $t \geq 0$. By the methods of the kinetic theory in [3], the time evolution of $f(\mathbf{w}, t)$ can be described as follows:

$$
\begin{aligned}
\frac{\partial f}{\partial t}(\mathbf{w}, t)= & \int_{\mathbb{R}^{m}} \int_{\mathbb{R}^{m} \times \mathbb{R}^{m}}\left[B_{\left(\left(' \mathbf{w}, \mathbf{w}_{*}\right) \rightarrow\left(\mathbf{w}, \mathbf{w}_{*}\right)\right)} \frac{1}{|J|} f\left({ }^{\prime} \mathbf{w}, t\right) f\left({ }^{\prime} \mathbf{w}_{*}, t\right)\right. \\
& \left.-B_{\left(\left(\mathbf{w}, \mathbf{w}_{*}\right) \rightarrow\left(\mathbf{w}^{\prime}, \mathbf{w}_{*}^{\prime}\right)\right)} f(\mathbf{w}, t) f\left(\mathbf{w}_{*}, t\right)\right] \mathrm{d} \mathbf{x} \mathrm{d} \mathbf{x}_{*} \mathrm{~d} \mathbf{w}_{*} .
\end{aligned}
$$

Here ' $\mathbf{w},{ }^{\prime} \mathbf{w}_{*}$ are pre-trading portfolios which generate $\mathbf{w}, \mathbf{w}_{*}$ after the trade, respectively. Applying the trading rule, we see that $\mathbf{w}=D(\mathbf{x}, \mathbf{r})\left({ }^{\prime} \mathbf{w}\right)+D(\mathbf{r})\left({ }^{\prime} \mathbf{w}_{*}\right)$ and that $\mathbf{w}_{*}=D\left(\mathbf{x}_{*}, \mathbf{r}\right)\left({ }^{\prime} \mathbf{w}_{*}\right)+D(\mathbf{r})\left({ }^{\prime} \mathbf{w}\right)$. In $(2.8),|J|$ is the absolute value of Jacobian from 
$\mathbf{w}, \mathbf{w}_{*}$ to ${ }^{\prime} \mathbf{w},{ }^{\prime} \mathbf{w}_{*}$. More precisely, $|J|=|D(\mathbf{x}, \mathbf{r})|\left|D\left(\mathbf{x}_{*}, \mathbf{r}\right)\right|-|D(\mathbf{r})|^{2}$ for any $\left(\mathbf{x}, \mathbf{x}_{*}\right)$. The kernels are given by

$$
\begin{aligned}
& B_{\left(\left({ }^{\prime} \mathbf{w}, \prime_{*}^{\prime}\right) \rightarrow\left(\mathbf{w}, \mathbf{w}_{*}\right)\right)}=\rho(\mathbf{x}) \rho\left(\mathbf{x}_{*}\right) \prod_{i=1}^{m} \mathbb{1}_{\left\{w_{i} \geq 0\right\}} \mathbb{1}_{\left\{w_{i *} \geq 0\right\}} \mathbb{1}_{\left\{{ }^{\prime} w_{i} \geq 0\right\}} \mathbb{1}_{\left\{{ }^{\prime} w_{i *} \geq 0\right\}}, \\
& B_{\left(\left(\mathbf{w}, \mathbf{w}_{*}\right) \rightarrow\left(\mathbf{w}^{\prime}, \mathbf{w}_{*}^{\prime}\right)\right)}=\rho(\mathbf{x}) \rho\left(\mathbf{x}_{*}\right) \prod_{i=1}^{m} \mathbb{1}_{\left\{w_{i} \geq 0\right\}} \mathbb{1}_{\left\{w_{i *} \geq 0\right\}} \mathbb{1}_{\left\{w_{i}^{\prime} \geq 0\right\}} \mathbb{1}_{\left\{w_{i *}^{\prime} \geq 0\right\}},
\end{aligned}
$$

where $\mathbb{1}_{\{\cdot \geq 0\}}$ is the characteristic function of the set $\{\cdot \geq 0\}$. Define

$$
\begin{aligned}
& Q^{+}(f, f)(\mathbf{w}, t)=\int_{\mathbb{R}^{m}} \int_{\mathbb{R}^{m} \times \mathbb{R}^{m}} B\left(\left({ }^{\prime} \mathbf{w}, \mathbf{w}_{*}\right) \rightarrow\left(\mathbf{w}, \mathbf{w}_{*}\right)\right) \\
& Q^{-}(f, f)(\mathbf{w}, t)=\int_{\mathbb{R}^{m}} \int_{\mathbb{R}^{m} \times \mathbb{R}^{m}} B\left(\left({ }^{\prime}, \mathbf{w}_{*}\right) \rightarrow\left(\mathbf{w}^{\prime}, \mathbf{w}_{*}^{\prime}\right)\right) \\
&
\end{aligned}
$$

If $Q^{ \pm}(f, f)(\mathbf{w}, t)$ are finite, then the decomposition

$$
Q(f, f)(\mathbf{w}, t)=Q^{+}(f, f)(\mathbf{w}, t)-Q^{-}(f, f)(\mathbf{w}, t)
$$

is meaningful. Letting $|\mathbf{w}|=\left(\sum_{i=1}^{m} w_{i}^{2}\right)^{1 / 2}$, we introduce a subclass of $L^{1}\left(\mathbb{R}^{m}\right)$

$$
L_{s}^{1}\left(\mathbb{R}^{m}\right)=\left\{f\left|f: \mathbb{R}^{m} \rightarrow \mathbb{R},\|f\|_{L_{s}^{1}}:=\int_{\mathbb{R}^{m}}\right| f(\mathbf{w}) \mid\left(1+|\mathbf{w}|^{2}\right)^{s / 2} \mathrm{~d} \mathbf{w}<\infty\right\}, \quad s \geq 0 .
$$

Definition 2.1 (mild solution). Given an initial datum $0 \leq f_{0} \in L^{1}\left(\mathbb{R}^{m}\right)$, we say that $f$ is a (global) mild solution of Equation (2.8) on $\mathbb{R}^{m} \times[0, \infty)$ with $\left.f\right|_{t=0}=f_{0}(\mathbf{w})$ if $f$ is nonnegative and satisfies the following:

(1) $f$ is measurable on $\mathbb{R}^{m} \times[0, \infty)$, and $f \in L^{\infty}\left([0, \infty) ; L^{1}\left(\mathbb{R}^{m}\right)\right)$.

(2) $\int_{0}^{t} Q^{ \pm}(f, f)(\mathbf{w}, \tau) \mathrm{d} \tau<\infty$, and

$$
f(\mathbf{w}, t)=f_{0}(\mathbf{w})+\int_{0}^{t} Q(f, f)(\mathbf{w}, \tau) \mathrm{d} \tau,
$$

where $\mathbf{w} \in \mathbb{R}^{m} \backslash Z, t \in[0, \infty)$, and $Z$ is a null set independent of $t$.

TheOREM 2.2. Suppose $0 \leq f_{0} \in L^{1}\left(\mathbb{R}^{m}\right)$. Then there exists a unique mild solution $f$ of (2.8) with conserved zeroth moment, such that $f \in C\left([0, \infty), L^{1}\left(\mathbb{R}^{m}\right)\right),\left.f\right|_{t=t_{0}}=f_{0}$. then

Furthermore, assume that $f_{0} \in L_{s}^{1}\left(\mathbb{R}^{m}\right)$ for some $s \geq 2$. If $E\left(\left|\zeta_{i}\right|^{s}\right)<\infty(1 \leq i \leq m)$,

$$
\int_{\mathbb{R}^{m}}|\mathbf{w}|^{s} f(\mathbf{w}, t) \mathrm{d} \mathbf{w} \leq e^{c t} \int_{\mathbb{R}^{m}}|\mathbf{w}|^{s} f_{0}(\mathbf{w}) \mathrm{d} \mathbf{w}, \quad t \geq 0,
$$

where $c$ is a constant depending only on $\sigma_{i}, r_{i}, s$, and $E\left(\left|\zeta_{i}\right|^{s}\right)$.

REMARK 2.3. We remark that the mild solution $f$ should actually be written as $f_{\mathbf{r}}$ to indicate the dependence on the parameter $\mathbf{r}=\left(r_{1}, \cdots, r_{m}\right)^{T}$. For simplicity, we will write $f(\mathbf{w}, t)$ instead of $f_{\mathbf{r}}(\mathbf{w}, t)$. 
Remark 2.4. Since debt is not permitted, we shall define $f_{0}(\mathbf{w})=0$ for $\mathbf{w} \notin \mathbb{R}_{+}^{m}$. Moreover, from (2.11) we can clearly see that

$$
f(\mathbf{w}, t)=0, \quad \forall \mathbf{w} \notin \mathbb{R}_{+}^{m}, \quad \forall t \in[0, \infty) .
$$

REMARK 2.5. In this paper, $c(m, s)$ only stands for a constant depending on $m$ and $s$. It may be different from line to line. So does $c(m)$.

\section{Proof of the Theorem 2.2.}

Part 1: Existence and uniqueness.

Proof. The proof of the existence and uniqueness of the solution is similar to that of the spatially homogeneous Boltzmann equation with bounded kernel (see [1] and [15]). First we prove that there exists $\delta>0$ depending on $\left\|f_{0}\right\|_{L^{1}}$ such that the solution exists on $\mathbb{R}^{m} \times[0, \delta]$. Then we extend the solution to $\mathbb{R}^{m} \times[0, \infty)$ by the conservation of the total number of agents (i.e. conservation of zeroth moment).

For $\delta>0$, let $\mathfrak{B}_{\delta}$ be a set of functions $f(\mathbf{w}, t)$ satisfying

(i) $f(\mathbf{w}, t)$ is measurable on $\mathbb{R}^{m} \times[0, \delta]$ and $f(\cdot, t) \in L^{1}\left(\mathbb{R}^{m}\right)$ for all $t \in[0, \delta]$;

(ii) for any $t \in[0, \delta], \mathbf{w} \mapsto f(\mathbf{w}, t)$ is measurable on $\mathbb{R}^{m}$;

(iii) $\|f\|_{\delta} \leq 2\left\|f_{0}\right\|_{L^{1}}$ with $\|f\|_{\delta}:=\sup _{t \in[0, \delta]}\|f(\cdot, t)\|_{L^{1}}$.

It is easy to check that $\left(\mathfrak{B}_{\delta},\|\cdot-\cdot\|_{\delta}\right)$ is a complete metric space. Moreover, we have for all $f, g \in \mathfrak{B}_{\delta}$ that

$$
\begin{gathered}
\int_{\mathbb{R}^{m}} Q^{ \pm}(|f|,|f|)(\mathbf{w}, t) \mathrm{d} \mathbf{w} \leq 4\left\|f_{0}\right\|_{L^{1}}^{2} \\
\int_{\mathbb{R}^{m}}|Q(|f|,|f|)(\mathbf{w}, t)-Q(|g|,|g|)(\mathbf{w}, t)| \mathrm{d} \mathbf{w} \leq 8\left\|f_{0}\right\|_{L^{1}}\|f-g\|_{\delta} .
\end{gathered}
$$

Define the operator

$$
\mathcal{T}(f)(\mathbf{w}, t)=f_{0}(\mathbf{w})+\int_{0}^{t} Q(|f|,|f|)(\mathbf{w}, \tau) \mathrm{d} \tau, \quad f \in \mathfrak{B}_{\delta}, \quad t \in[0, \delta] .
$$

Then we have

$$
\|\mathcal{T}(f)\|_{\delta}:=\sup _{t \in[0, \delta]}\|\mathcal{T}(f)(\cdot, t)\|_{L^{1}} \leq\left\|f_{0}\right\|_{L^{1}}+8\left\|f_{0}\right\|_{L^{1}}^{2} \delta
$$

and

$$
\|\mathcal{T}(f)-\mathcal{T}(g)\|_{\delta} \leq 8\left\|f_{0}\right\|_{L^{1}}\|f-g\|_{\delta} \delta
$$

Choose $\delta$ small enough such that $0<\delta<\frac{1}{8\left\|f_{0}\right\|_{L^{1}}}$. Thus $\mathcal{T}$ is a contractive mapping from $\mathfrak{B}_{\delta}$ into itself. This implies that $\mathcal{T}$ has a unique fixed point $f \in \mathfrak{B}_{\delta}$, i.e.

$$
f(\mathbf{w}, t)=f_{0}(\mathbf{w})+\int_{0}^{t} Q(|f|,|f|)(\mathbf{w}, \tau) \mathrm{d} \tau, \quad \mathbf{w} \in \mathbb{R}^{m} \backslash Z_{t}, \quad t \in[0, \delta],
$$

where $Z_{t} \in \mathbb{R}^{m}$ is a null set depending on $t$, such that

$$
\int_{0}^{t} Q^{ \pm}(|f|,|f|)(\mathbf{w}, \tau) \mathrm{d} \tau<\infty, \quad \mathbf{w} \in R^{m} \backslash Z_{t} .
$$


Now we focus on proving the nonnegativity of $f(\mathbf{w}, t)$. Denote $(f(\mathbf{w}, t))^{+}=$ $\max \{f(\mathbf{w}, t), 0\}$. Then $\forall \mathbf{w} \in \mathbb{R}^{m} \backslash Z_{t}$,

$$
(-f(\mathbf{w}, t))^{+} \leq \int_{0}^{t} Q^{-}(|f|,|f|)(\mathbf{w}, \tau) \mathbb{1}_{\{f(\mathbf{w}, \tau) \leq 0\}} \mathrm{d} \tau .
$$

This combined with the fact $(-f(\mathbf{w}, t))^{+}=|f| \mathbb{1}_{\{f(\mathbf{w}, t) \leq 0\}}$ shows that

$$
\begin{aligned}
\left\|(-f(\cdot, t))^{+}\right\|_{L^{1}} & \leq \int_{\mathbb{R}^{m}} \int_{0}^{t} Q^{-}(|f|,|f|)(\mathbf{w}, \tau) \mathbb{1}_{\{f(\mathbf{w}, \tau) \leq 0\}} \mathrm{d} \tau \mathrm{d} \mathbf{w} \\
& \leq 2\left\|f_{0}\right\|_{L^{1}} \int_{0}^{t}\left\|(-f(\cdot, \tau))^{+}\right\|_{L^{1}} \mathrm{~d} \tau, \quad t \in[0, \delta] .
\end{aligned}
$$

Thus, by the Gronwall inequality, $\left\|(-f(\cdot, t))^{+}\right\|_{L^{1}}=0$ for all $t \in[0, \delta]$. Equivalently, $\forall t \in[0, \delta], f(\mathbf{w}, t) \geq 0$ for a.e. $\mathbf{w} \in \mathbb{R}^{m}$. Furthermore, $f$ can be modified by redefining the functions on a null set such that it satisfies (2.11). In fact, let $\tilde{f}(\mathbf{w}, t)=|\mathcal{T}(f)(\mathbf{w}, t)|$. Then for all $\mathbf{w} \in \mathbb{R}^{m}, \tilde{f}$ is nonnegative, continuous with respect to $t \in[0, \delta]$, and satisfies $\|\tilde{f}-\mathcal{T}(\tilde{f})\|_{\delta}=0$. Meanwhile, the continuity of $\tilde{f}$ in $t$ implies that $Z_{t}$ can be modified with a null set independent of $t$, which is denoted by $Z$. Then $\tilde{f}$ is a local mild solution on $\mathbb{R}^{m} \times[0, \delta]$. In the following, denote $\tilde{f}$ by $f$ for simplicity.

It is clear that the $L^{1}$-norm of the solution is preserved: $\|f(\cdot, \delta)\|_{L^{1}}=\left\|f_{0}\right\|_{L^{1}}$. Since $\delta$ depends only on $\left\|f_{0}\right\|_{L^{1}}$, we can extend the solution to an arbitrary time. Indeed, applying the above argument and replacing the initial datum $f_{0}$ by $f(\cdot, \delta)$, we can repeat the procedure up to time $2 \delta$, and so on. Then the extended function $f$ is a unique solution and belongs to $C\left([0, \infty), L^{1}\left(\mathbb{R}^{m}\right)\right)$ with $\|f(\cdot, t)\|_{L^{1}}=\left\|f_{0}\right\|_{L^{1}}$.

Part 2: Proof of (2.12). Suppose $f_{0} \in L_{s}^{1}\left(\mathbb{R}^{m}\right)(s \geq 2)$. By the density scaling and the zeroth moment conservation, we may assume the initial datum to be normalized: $\int_{\mathbb{R}^{m}} f_{0}(\mathbf{w}) \mathrm{d} \mathbf{w}=1$. Let $\varphi_{n}(\mathbf{w})=\left(1+|\mathbf{w}|^{2}\right)_{n}^{s / 2}=\min \left\{\left(1+|\mathbf{w}|^{2}\right)^{s / 2}, n\right\}$. Then we get

$$
\int_{0}^{t} \mathrm{~d} \tau \int_{\mathbb{R}^{m}} Q^{-}(f, f)(\mathbf{w}, \tau) \varphi_{n}(\mathbf{w}) \mathrm{d} \mathbf{w} \leq \int_{0}^{t}\left\|f \varphi_{n}\right\|_{L^{1}} \mathrm{~d} \tau
$$

and

$$
\begin{aligned}
& \int_{0}^{t} \mathrm{~d} \tau \int_{\mathbb{R}^{m}} Q^{+}(f, f)(\mathbf{w}, \tau) \varphi_{n}(\mathbf{w}) \mathrm{d} \mathbf{w} \\
\leq & \int_{0}^{t} \mathrm{~d} \tau \int_{\mathbb{R}^{m} \times \mathbb{R}^{m}} \int_{\mathbb{R}^{m} \times \mathbb{R}^{m}} \rho(\mathbf{x}) \rho\left(\mathbf{x}_{*}\right) \varphi_{n}\left(\mathbf{w}^{\prime}\right) f(\mathbf{w}, \tau) f\left(\mathbf{w}_{*}, \tau\right) \mathrm{d} \mathbf{x} \mathrm{d} \mathbf{x}_{*} \mathrm{~d} \mathbf{w}_{*} \mathrm{~d} \mathbf{w} .
\end{aligned}
$$

It is easily seen that when $a_{i}, b_{i} \geq 0$,

$$
a_{1} \leq a_{2}, b_{1} \leq b_{2} \Rightarrow a_{1} \wedge b_{1} \leq a_{2} \wedge b_{2}, \quad\left(\sum_{i=1}^{l} a_{i}\right) \wedge\left(\sum_{j=1}^{m} b_{j}\right) \leq \sum_{i=1}^{l} \sum_{j=1}^{m}\left(a_{i} \wedge b_{j}\right) .
$$

Moreover, if $a \geq 1, b \geq 0$, then $(a \cdot b) \wedge n \leq a(b \wedge n)$. These inequalities together with

$$
\left(w_{i}^{\prime}\right)^{2} \leq 4\left(w_{i}^{2}+w_{i *}^{2}+x_{i}^{2} w_{i}^{2}\right)
$$

imply that

$$
\varphi_{n}\left(\mathbf{w}^{\prime}\right) \leq c(m, s)\left[\varphi_{n}(\mathbf{w})+\varphi_{n}\left(\mathbf{w}_{*}\right)+\sum_{i=1}^{m}\left(\left|x_{i}\right|^{s}+1\right) \varphi_{n}(\mathbf{w})\right] .
$$


Substituting this inequality into (2.22), we get

$$
\int_{0}^{t} \mathrm{~d} \tau \int_{\mathbb{R}^{m}} Q^{+}(f, f)(\mathbf{w}, \tau) \varphi_{n}(\mathbf{w}) \mathrm{d} \mathbf{w} \leq c(m, s)\left(\sum_{i=1}^{m} \sigma_{i}^{s} E\left(\left|\zeta_{i}\right|^{s}\right)+m+2\right) \int_{0}^{t}\left\|f \varphi_{n}\right\|_{L^{1}} \mathrm{~d} \tau .
$$

From this and (2.21), by the Gronwall inequality we obtain the bound

$$
\left\|f(\cdot, t) \varphi_{n}\right\|_{L^{1}} \leq\left\|f_{0}\right\|_{L_{s}^{1}} \exp \{C t\}, \quad t \geq 0
$$

where $C$ is a constant independent of $n: C=c(m, s)\left(\sum_{i=1}^{m} \sigma_{i}^{s} E\left(\left|\zeta_{i}\right|^{s}\right)+m+2\right)+1$. Letting $n \rightarrow \infty$, we see that $f \in L_{l o c}^{\infty}\left([0, \infty) ; L_{s}^{1}\left(\mathbb{R}^{m}\right)\right)$. Therefore, multiplying both sides of (2.11) by $|\mathbf{w}|^{s}$ and integrating with respect to $\mathbf{w}$ on $\mathbb{R}^{m}$, we obtain

$$
\begin{aligned}
& \int_{\mathbb{R}^{m}}|\mathbf{w}|^{s} f(\mathbf{w}, t) \mathrm{d} \mathbf{w} \\
& =\int_{\mathbb{R}^{m}}|\mathbf{w}|^{s} f_{0}(\mathbf{w}) \mathrm{d} \mathbf{w}+\int_{0}^{t} \int_{\mathbb{R}^{m} \times \mathbb{R}^{m}} \int_{\mathbb{R}^{m} \times \mathbb{R}^{m}} \rho(\mathbf{x}) \rho\left(\mathbf{x}_{*}\right) \prod_{i=1}^{m} \mathbb{1}_{\left\{w_{i} \geq 0\right\}} \mathbb{1}_{\left\{w_{i *} \geq 0\right\}} \\
& \quad \times\left(1-\prod_{i=1}^{m} \mathbb{1}_{\left\{w_{i}^{\prime} \geq 0\right\}} \mathbb{1}_{\left\{w_{i *}^{\prime} \geq 0\right\}}\right) f(\mathbf{w}, \tau) f\left(\mathbf{w}_{*}, \tau\right)\left(|\mathbf{w}|^{s}-\left|\mathbf{w}^{\prime}\right|^{s}\right) \mathrm{d} \mathbf{x} \mathrm{d} \mathbf{x}_{*} \mathrm{~d} \mathbf{w} \mathrm{d} \mathbf{w}_{*} \mathrm{~d} \tau \\
& \quad+\int_{0}^{t} \int_{\mathbb{R}_{+}^{m} \times \mathbb{R}_{+}^{m}} \int_{\mathbb{R}^{m} \times \mathbb{R}^{m}} \rho(\mathbf{x}) \rho\left(\mathbf{x}_{*}\right) f(\mathbf{w}, \tau) f\left(\mathbf{w}_{*}, \tau\right)\left(\left|\mathbf{w}^{\prime}\right|^{s}-|\mathbf{w}|^{s}\right) \mathrm{d} \mathbf{x} \mathrm{d} \mathbf{x}_{*} \mathrm{~d} \mathbf{w} \mathrm{d} \mathbf{w}_{*} \mathrm{~d} \tau \\
& =: \int_{\mathbb{R}^{m}}|\mathbf{w}|^{s} f_{0}(\mathbf{w}) \mathrm{d} \mathbf{w}+A_{1}+A_{2} .
\end{aligned}
$$

(i) ( The case of $s=2)$. By (2.1)-(2.2), one easily sees that

$$
1-\prod_{i=1}^{m} \mathbb{1}_{\left\{w_{i}^{\prime} \geq 0\right\}} \mathbb{1}_{\left\{w_{i *}^{\prime} \geq 0\right\}} \leq \sum_{i=1}^{m} \mathbb{1}_{\left\{x_{i}<r_{i}-1\right\}}+\sum_{i=1}^{m} \mathbb{1}_{\left\{x_{i *}<r_{i}-1\right\}} .
$$

Since

$$
\begin{aligned}
& \int_{0}^{t} \mathrm{~d} \tau \int_{\mathbb{R}^{m} \times \mathbb{R}^{m}} \mathbb{1}_{\left\{x_{i}<r_{i}-1\right\}} \rho(\mathbf{x}) \rho\left(\mathbf{x}_{*}\right) \mathrm{d} \mathbf{x} \mathrm{d} \mathbf{x}_{*} \int_{\mathbb{R}_{+}^{m} \times \mathbb{R}_{+}^{m}}|\mathbf{w}|^{2} f(\mathbf{w}, \tau) f\left(\mathbf{w}_{*}, \tau\right) \mathrm{d} \mathbf{w}_{*} \mathrm{~d} \mathbf{w} \\
\leq & \int_{0}^{t} \mathrm{~d} \tau \int_{\mathbb{R}^{m} \times \mathbb{R}^{m}} \rho(\mathbf{x}) \rho\left(\mathbf{x}_{*}\right) \frac{\left|x_{i}\right|^{2}}{\left(1-r_{i}\right)^{2}} \mathrm{~d} \mathbf{x} \mathrm{d} \mathbf{x}_{*} \int_{\mathbb{R}_{+}^{m} \times \mathbb{R}_{+}^{m}}|\mathbf{w}|^{2} f(\mathbf{w}, \tau) f\left(\mathbf{w}_{*}, \tau\right) \mathrm{d} \mathbf{w}_{*} \mathrm{~d} \mathbf{w} \\
\leq & \frac{\sigma_{i}^{2}}{\left(1-r_{i}\right)^{2}} \int_{0}^{t} \mathrm{~d} \tau \int_{\mathbb{R}^{m}}|\mathbf{w}|^{2} f(\mathbf{w}, \tau) \mathrm{d} \mathbf{w}
\end{aligned}
$$

it follows from the distribution of $\boldsymbol{\eta}, \boldsymbol{\eta}_{*}$ that

$$
A_{1} \leq \sum_{i=1}^{m} \frac{2 \sigma_{i}^{2}}{\left(1-r_{i}\right)^{2}} \int_{0}^{t} \mathrm{~d} \tau \int_{\mathbb{R}^{m}}|\mathbf{w}|^{2} f(\mathbf{w}, \tau) \mathrm{d} \mathbf{w} .
$$

In the following we will denote

$$
|\mathbf{r}|=\max _{1 \leq i \leq m}\left\{r_{i}\right\} .
$$


By (2.1)-(2.5), a simple calculation shows that

$$
A_{2} \leq c(m)\left(|\mathbf{r}|+|\mathbf{r}|^{2}+\sum_{i=1}^{m} \sigma_{i}^{2}\right) \int_{0}^{t} \mathrm{~d} \tau \int_{\mathbb{R}^{m}}|\mathbf{w}|^{2} f(\mathbf{w}, \tau) \mathrm{d} \mathbf{w} .
$$

Let

$$
c=\sum_{i=1}^{m} \frac{2 \sigma_{i}^{2}}{\left(1-r_{i}\right)^{2}}+c(m)\left(|\mathbf{r}|+|\mathbf{r}|^{2}+\sum_{i=1}^{m} \sigma_{i}^{2}\right) .
$$

Combining (2.30) and (2.32), we get (2.12) using Gronwall inequality.

(ii) (The case of $s>2$ ). The same argument as that in (2.29) shows that

$$
A_{1} \leq \sum_{i=1}^{m} \frac{2 \sigma_{i}^{2}}{\left(1-r_{i}\right)^{2}} \int_{0}^{t} \mathrm{~d} \tau \int_{\mathbb{R}^{m}}|\mathbf{w}|^{s} f(\mathbf{w}, \tau) \mathrm{d} \mathbf{w}
$$

Since

$$
\nabla|\mathbf{w}|^{s}=s|\mathbf{w}|^{s-2} \mathbf{w}, \quad H_{|\cdot| s}(\mathbf{w})=s(s-2)|\mathbf{w}|^{s-4} \mathbf{w} \mathbf{w}^{T}+s|\mathbf{w}|^{s-2} \mathbf{I},
$$

it follows by Taylor's formula that

$$
\left|\mathbf{w}^{\prime}\right|^{s}-|\mathbf{w}|^{s}=\left\langle\nabla|\mathbf{w}|^{s}, \mathbf{w}^{\prime}-\mathbf{w}\right\rangle+\frac{s(s-2)}{2}|\tilde{\mathbf{w}}|^{s-4}\left\langle\mathbf{w}^{\prime}-\mathbf{w}, \tilde{\mathbf{w}}\right\rangle^{2}+\frac{s}{2}|\tilde{\mathbf{w}}|^{s-2}\left|\mathbf{w}^{\prime}-\mathbf{w}\right|^{2},
$$

where $\tilde{\mathbf{w}}=\mathbf{w}+\theta\left(\mathbf{w}^{\prime}-\mathbf{w}\right), \theta \in(0,1)$, and $\langle\cdot, \cdot\rangle$ denotes the inner product in $\mathbb{R}^{m}$. Substituting the identity (2.35) into $A_{2}$ and splitting the integration $A_{2}$ into two parts $A_{21}+A_{22}$, we get

$$
\begin{aligned}
A_{21}= & \int_{0}^{t} \mathrm{~d} \tau \int_{\mathbb{R}_{+}^{m} \times \mathbb{R}_{+}^{m}} \int_{\mathbb{R}^{m} \times \mathbb{R}^{m}} \rho(\mathbf{x}) \rho\left(\mathbf{x}_{*}\right) \\
& \times s|\mathbf{w}|^{s-2}\left\langle\mathbf{w}, \mathbf{w}^{\prime}-\mathbf{w}\right\rangle f(\mathbf{w}, \tau) f\left(\mathbf{w}_{*}, \tau\right) \mathrm{d} \mathbf{x} \mathrm{d} \mathbf{x}_{*} \mathrm{~d} \mathbf{w} \mathrm{d} \mathbf{w}_{*} \\
\leq & \leq s|\mathbf{r}| \int_{0}^{t} \mathrm{~d} \tau \int_{\mathbb{R}_{+}^{m} \times \mathbb{R}_{+}^{m}}|\mathbf{w}|^{s-2}\left\langle\mathbf{w}, \mathbf{w}_{*}\right\rangle f(\mathbf{w}, \tau) f\left(\mathbf{w}_{*}, \tau\right) \mathrm{d} \mathbf{w} \mathrm{d} \mathbf{w}_{*} .
\end{aligned}
$$

Moreover, by the Cauchy-Schwarz inequality and Hölder's inequality we have

$$
\begin{aligned}
& \int_{\mathbb{R}_{+}^{m} \times \mathbb{R}_{+}^{m}}|\mathbf{w}|^{s-2}\left\langle\mathbf{w}, \mathbf{w}_{*}\right\rangle f(\mathbf{w}, \tau) f\left(\mathbf{w}_{*}, \tau\right) \mathrm{d} \mathbf{w} \mathrm{d} \mathbf{w}_{*} \\
\leq & \int_{\mathbb{R}_{+}^{m}}|\mathbf{w}|^{s-1} f(\mathbf{w}, \tau) \mathrm{d} \mathbf{w} \int_{\mathbb{R}_{+}^{m}}\left|\mathbf{w}_{*}\right| f\left(\mathbf{w}_{*}, \tau\right) \mathrm{d} \mathbf{w}_{*} \leq \int_{\mathbb{R}^{m}}|\mathbf{w}|^{s} f(\mathbf{w}, \tau) \mathrm{d} \mathbf{w},
\end{aligned}
$$

which gives

$$
A_{21} \leq s|\mathbf{r}| \int_{0}^{t} \mathrm{~d} \tau \int_{\mathbb{R}^{m}}|\mathbf{w}|^{s} f(\mathbf{w}, \tau) \mathrm{d} \mathbf{w} .
$$


Using the Cauchy-Schwarz inequality and Hölder's inequality again, we have

$$
\begin{aligned}
& A_{22}= \int_{0}^{t} \mathrm{~d} \tau \int_{\mathbb{R}_{+}^{m} \times \mathbb{R}_{+}^{m}} f(\mathbf{w}, \tau) f\left(\mathbf{w}_{*}, \tau\right) \mathrm{d} \mathbf{w} \mathrm{d} \mathbf{w}_{*} \int_{\mathbb{R}^{m} \times \mathbb{R}^{m}} \rho(\mathbf{x}) \rho\left(\mathbf{x}_{*}\right) \\
& \times\left(\frac{s(s-2)}{2}|\tilde{\mathbf{w}}|^{s-4}\left\langle\mathbf{w}^{\prime}-\mathbf{w}, \tilde{\mathbf{w}}\right\rangle^{2}+\frac{s}{2}|\tilde{\mathbf{w}}|^{s-2}\left|\mathbf{w}^{\prime}-\mathbf{w}\right|^{2}\right) \mathrm{d} \mathbf{x} \mathrm{d} \mathbf{x}_{*} \\
& \leq \frac{s(s-1)}{2} \int_{0}^{t} \mathrm{~d} \tau \int_{\mathbb{R}_{+}^{m} \times \mathbb{R}_{+}^{m}} f(\mathbf{w}, \tau) f\left(\mathbf{w}_{*}, \tau\right) \mathrm{d} \mathbf{w} \mathrm{d} \mathbf{w}_{*} \int_{\mathbb{R}^{m} \times \mathbb{R}^{m}} \rho(\mathbf{x}) \\
& \times \rho\left(\mathbf{x}_{*}\right)|\tilde{\mathbf{w}}|^{s-2}\left|\mathbf{w}^{\prime}-\mathbf{w}\right|^{2} \mathrm{~d} \mathbf{x} \mathrm{d} \mathbf{x}_{*} \\
& \leq c(s) \int_{0}^{t} \mathrm{~d} \tau \int_{\mathbb{R}_{+}^{m} \times \mathbb{R}_{+}^{m}} f(\mathbf{w}, \tau) f\left(\mathbf{w}_{*}, \tau\right) \mathrm{d} \mathbf{w} \mathrm{d} \mathbf{w}_{*} \int_{\mathbb{R}^{m} \times \mathbb{R}^{m}} \rho(\mathbf{x}) \rho\left(\mathbf{x}_{*}\right) \\
& \quad \times\left(\left|\mathbf{w}^{\prime}-\mathbf{w}\right|^{s}+\left|\mathbf{w}^{\prime}-\mathbf{w}\right|^{2}|\mathbf{w}|^{s-2}\right) \mathrm{d} \mathbf{x} \mathrm{d} \mathbf{x}_{*} \\
& \leq c(m, s)\left[|\mathbf{r}|^{2}+|\mathbf{r}|^{s}+\sum_{i=1}^{m} \sigma_{i}^{2}+\sum_{i=1}^{m} \sigma_{i}^{s} E\left(\left|\zeta_{i}\right|^{s}\right)\right] \int_{0}^{t} \mathrm{~d} \tau \int_{\mathbb{R}^{m}}|\mathbf{w}|^{s} f(\mathbf{w}, \tau) \mathrm{d} \mathbf{w}
\end{aligned}
$$

Hence

$$
A_{2} \leq\left(s|\mathbf{r}|+c(m, s)\left[|\mathbf{r}|^{2}+|\mathbf{r}|^{s}+\sum_{i=1}^{m} \sigma_{i}^{2}+\sum_{i=1}^{m} \sigma_{i}^{s} E\left(\left|\zeta_{i}\right|^{s}\right)\right]\right) \int_{0}^{t} \mathrm{~d} \tau \int_{\mathbb{R}^{m}}|\mathbf{w}|^{s} f(\mathbf{w}, \tau) \mathrm{d} \mathbf{w} .
$$

Let

$$
c=\sum_{i=1}^{m} \frac{2 \sigma_{i}^{2}}{\left(1-r_{i}\right)^{2}}+s|\mathbf{r}|+c(m, s)\left[|\mathbf{r}|^{2}+|\mathbf{r}|^{s}+\sum_{i=1}^{m} \sigma_{i}^{2}+\sum_{i=1}^{m} \sigma_{i}^{s} E\left(\left|\zeta_{i}\right|^{s}\right)\right] .
$$

Combining (2.34) and (2.40), we get (2.12) for any $s>2$ by using Gronwall inequality

If the assumption on the initial datum is strengthened, the following result can be obtained.

TheOREm 2.6. Suppose that $f_{0} \in L^{1}\left(\mathbb{R}^{m}\right) \cap L^{2}\left(\mathbb{R}^{m}\right)$. Then the unique mild solution $f$ of $(2.8)$ belongs to $C\left([0, \infty), L^{1}\left(\mathbb{R}^{m}\right)\right)$ and satisfies

$$
\begin{aligned}
f^{2}(\mathbf{w}, t)= & f_{0}^{2}(\mathbf{w})+2 \int_{0}^{t} \int_{\mathbb{R}^{m}} \int_{\mathbb{R}^{m} \times \mathbb{R}^{m}}\left[B_{\left.\left({ }^{\prime} \mathbf{w}, \mathbf{w}_{*}\right) \rightarrow\left(\mathbf{w}, \mathbf{w}_{*}\right)\right)} \frac{1}{|J|} f\left({ }^{\prime} \mathbf{w}, \tau\right)\right. \\
& \left.\times f\left({ }^{\prime} \mathbf{w}_{*}, \tau\right) f(\mathbf{w}, \tau)-B{ }_{\left(\left(\mathbf{w}, \mathbf{w}_{*}\right) \rightarrow\left(\mathbf{w}^{\prime}, \mathbf{w}_{*}^{\prime}\right)\right)} f^{2}(\mathbf{w}, \tau) f\left(\mathbf{w}_{*}, \tau\right)\right] \mathrm{d} \mathbf{x} \mathrm{d} \mathbf{x}_{*} \mathrm{~d} \mathbf{w}_{*} \mathrm{~d} \tau,
\end{aligned}
$$

where $\mathbf{w} \in \mathbb{R}^{m} \backslash Z, t \in[0, \infty)$, and $Z$ is a null set independent of $t$.

Proof. The proof is essentially the same as that for the previous one. First we shall prove that there exists $\varepsilon>0$ depending on $\left\|f_{0}\right\|_{L^{1}}$ such that the statement holds on $\mathbb{R}^{m} \times[0, \varepsilon]$. Let $\mathfrak{M}_{\varepsilon}$ be a set of functions $f(\mathbf{w}, t)$ satisfying:

(i) $f(\mathbf{w}, t)$ is measurable on $\mathbb{R}^{m} \times[0, \varepsilon]$;

(ii) for any $t \in[0, \varepsilon], \mathbf{w} \mapsto f(\mathbf{w}, t)$ is measurable on $\mathbb{R}^{m}$; 
(iii) $\|f\|_{\varepsilon}:=\sup _{t \in[0, \varepsilon]}\|f(\cdot, t)\|_{L^{1}} \leq 2\left\|f_{0}\right\|_{L^{1}}, \sup _{t \in[0, \varepsilon]}\|f(\cdot, t)\|_{L^{2}} \leq 2\left\|f_{0}\right\|_{L^{2}}$.

We observe that $\left(\mathfrak{M}_{\varepsilon},\|\cdot-\cdot\|_{\varepsilon}\right)$ is a complete metric space. Let $\mathcal{T}$ be the same operator as that in (2.15):

$$
\mathcal{T}(f)(\mathbf{w}, t)=f_{0}(\mathbf{w})+\int_{0}^{t} Q(|f|,|f|)(\mathbf{w}, \tau) \mathrm{d} \tau, \quad f \in \mathfrak{M}_{\varepsilon}, \quad t \in[0, \varepsilon] .
$$

Then for all $f, g \in \mathfrak{M}_{\varepsilon}$, the same argument as that for Theorem 2.2 shows that

$$
\|\mathcal{T}(f)\|_{\varepsilon} \leq\left\|f_{0}\right\|_{L^{1}}+8\left\|f_{0}\right\|_{L^{1}}^{2} \varepsilon, \quad\|\mathcal{T}(f)-\mathcal{T}(g)\|_{\varepsilon} \leq 8\left\|f_{0}\right\|_{L^{1}}\|f-g\|_{\varepsilon} \varepsilon .
$$

Now we estimate $\|\mathcal{T}(f)(\cdot, t)\|_{L^{2}}$. Let $\psi \in L^{\infty}\left(\mathbb{R}^{m}\right) \bigcap L^{2}\left(\mathbb{R}^{m}\right)$. By the Hölder inequality we have

$$
\int_{\mathbb{R}^{m}} Q^{-}(|f|,|f|)(\mathbf{w}, t) \psi(\mathbf{w}) \mathrm{d} \mathbf{w} \leq 4\left\|f_{0}\right\|_{L^{1}}\left\|f_{0}\right\|_{L^{2}}\|\psi\|_{L^{2}} .
$$

Moreover, using a change of variables, we obtain

$$
\begin{aligned}
& \int_{\mathbb{R}^{m}} Q^{+}(|f|,|f|)(\mathbf{w}, t) \psi(\mathbf{w}) \mathrm{d} \mathbf{w} \\
&= \int_{\mathbb{R}^{m} \times \mathbb{R}^{m}} \int_{\mathbb{R}^{m} \times \mathbb{R}^{m}} \rho(\mathbf{x}) \rho\left(\mathbf{x}_{*}\right) \prod_{i=1}^{m} \mathbb{1}_{\left\{w_{i}^{\prime} \geq 0\right\}} \mathbb{1}_{\left\{w_{i *}^{\prime} \geq 0\right\}} \mathbb{1}_{\left\{w_{i} \geq 0\right\}} \mathbb{1}_{\left\{w_{i *} \geq 0\right\}} \\
& \times \psi\left(\mathbf{w}^{\prime}\right)\left|f(\mathbf{w}, t) \| f\left(\mathbf{w}_{*}, t\right)\right| \mathrm{d} \mathbf{w}_{*} \mathrm{~d} \mathbf{w} \mathrm{d} \mathbf{x} \mathrm{d} \mathbf{x}_{*} \\
& \leq \frac{1}{\prod_{i=1}^{m} r_{i}} \int_{\mathbb{R}^{m}} \rho(\mathbf{x}) \mathrm{d} \mathbf{x} \int_{\mathbb{R}^{m} \times \mathbb{R}^{m}}\left|\psi\left(\mathbf{w}_{*}\right)\right||f(\mathbf{w}, t)| \\
& \quad \times\left|f\left(\frac{w_{1 *}-\left(1-r_{1}+x_{1}\right) w_{1}}{r_{1}}, \cdots, \frac{w_{m *}-\left(1-r_{m}+x_{m}\right) w_{m}}{r_{m}}, t\right)\right| \mathrm{d} \mathbf{w}_{*} \mathrm{~d} \mathbf{w} \\
& \leq
\end{aligned}
$$

As a consequence of (2.43)-(2.44), we have

$$
\langle\mathcal{T}(f), \psi\rangle \leq\left\|f_{0}\right\|_{L^{2}}\|\psi\|_{L^{2}}+4\left(1+\left(\prod_{i=1}^{m} r_{i}\right)^{-\frac{1}{2}}\right) \int_{0}^{t}\left\|f_{0}\right\|_{L^{1}}\left\|f_{0}\right\|_{L^{2}}\|\psi\|_{L^{2}} \mathrm{~d} \tau .
$$

Thus

$$
\sup _{0 \leq t \leq \varepsilon}\|\mathcal{T}(f)(\cdot, t)\|_{L^{2}} \leq\left\|f_{0}\right\|_{L^{2}}+4\left(1+\left(\prod_{i=1}^{m} r_{i}\right)^{-\frac{1}{2}}\right)\left\|f_{0}\right\|_{L^{1}}\left\|f_{0}\right\|_{L^{2} \varepsilon}
$$

Choosing $\varepsilon=\delta=\frac{\sqrt{\prod_{i=1}^{m} r_{i}}}{8\left(\sqrt{\prod_{i=1}^{m} r_{i}}+1\right)}\left\|f_{0}\right\|_{L^{1}}\left(<1 / 8\left\|f_{0}\right\|_{L^{1}}\right)$ with $\delta$ given in Theorem 2.2, we see that $\mathcal{T}$ is a contractive mapping from $\mathfrak{M}_{\varepsilon}$ into itself. Thus by the same argument as that in Theorem 2.2, there exists a local unique mild solution $f$ of (2.8) such that (2.42) holds on $\mathbb{R}^{m} \times[0, \varepsilon]$. Moreover, since $\varepsilon$ depends only on $\left\|f_{0}\right\|_{L^{1}}, f$ can also be inductively extended to be the unique mild solution of (2.8) satisfying (2.42). 


\section{Time scaled solutions and some estimates}

It is quite difficult to study the large time behavior of $f$ since $f$ conserves only the zeroth moment. However, a feasible method dealing with the problem has been used previously for the one-dimensional model in [8] and [9]. The analysis, which is similar to the quasi-elastic limit of granular gas [23], is to some extent analogous to that for the approximation of the Boltzmann equation by the Landau equation when grazing collision is taken into consideration [24]. This section is devoted to proving several lemmas which are needed to establish the main theorem in the next section.

Recall that $|\mathbf{r}|=\max _{1 \leq i \leq 1}\left\{r_{i}\right\}$. If we set $t^{\prime}=|\mathbf{r}| t$, a new probability density $g_{\mathbf{r}}\left(\mathbf{w}, t^{\prime}\right)=$ $g_{\mathbf{r}}(\mathbf{w},|\mathbf{r}| t)=f(\mathbf{w}, t)$ is then introduced. It is clear that $g(\mathbf{w}, 0)=f(\mathbf{w}, 0)$ and

$$
\int_{\mathbb{R}^{m}} g_{\mathbf{r}}\left(\mathbf{w}, t^{\prime}\right) \mathrm{d} \mathbf{w}=\int_{\mathbb{R}^{m}} f(\mathbf{w}, t) \mathrm{d} \mathbf{w}=\int_{\mathbb{R}^{m}} f_{0}(\mathbf{w}) \mathrm{d} \mathbf{w} .
$$

Furthermore, fixing $t^{\prime}$ and taking $\sigma_{i}^{2}=\lambda_{i} r_{i}$, we obtain from (2.12) that

$$
\int_{\mathbb{R}^{m}}|\mathbf{w}|^{s} g_{\mathbf{r}}\left(\mathbf{w}, t^{\prime}\right) \mathrm{d} \mathbf{w} \leq \exp \left(c t^{\prime} /|\mathbf{r}|\right) \int_{\mathbb{R}^{m}}|\mathbf{w}|^{s} g(\mathbf{w}, 0) \mathrm{d} \mathbf{w}
$$

where $c$ is the constant given in (2.33) for $s=2$ and in (2.41) for $s>2$. The explicit formulas of $c$ show that $c /|\mathbf{r}|$ is bounded above with a constant independent of $\mathbf{r}$. This gives the boundedness of $\int_{\mathbb{R}^{m}}|\mathbf{w}|^{s} g_{\mathbf{r}}\left(\mathbf{w}, t^{\prime}\right) \mathrm{d} \mathbf{w}$ for any fixed $t^{\prime}$.

REMARK 3.1. By the density scaling and the zeroth moment conservation, we may assume $\int_{\mathbb{R}^{m}} f(\mathbf{w}, 0) \mathrm{d} \mathbf{w}=1$, which implies $\int_{\mathbb{R}^{m}} g_{\mathbf{r}}\left(\mathbf{w}, t^{\prime}\right) \mathrm{d} \mathbf{w}=1$. Additionally, in Section 3 and Section 4 we simply write $g_{\mathbf{r}}(\mathbf{w}, t)$ instead of $g_{\mathbf{r}}\left(\mathbf{w}, t^{\prime}\right)$ for convenience.

With these preparations, we turn to the proof of our result. It is easy to prove the following lemma by an argument similar to that for (2.12).

Lemma 3.2. Given $g(\cdot, 0) \in L_{2}^{1}\left(\mathbb{R}^{m}\right)$ and positive constants $\lambda_{i}(1 \leq i \leq m)$, we suppose that $\sigma_{i}^{2}=\lambda_{i} r_{i}$ and (2.5)-(2.6) hold. Then

$$
\lim _{|\mathbf{r}| \rightarrow 0^{+}}\left|\int_{\mathbb{R}^{m}} \mathbf{w} g_{\mathbf{r}}(\mathbf{w}, t) \mathrm{d} \mathbf{w}-\int_{\mathbb{R}^{m}} \mathbf{w} g(\mathbf{w}, 0) \mathrm{d} \mathbf{w}\right|=0, \quad \forall t \geq 0 .
$$

Proof. Without loss of generality, we may assume $0<|\mathbf{r}| \leq \frac{1}{2}$. We obtain the following equation immediately from (2.11) and the definition of $g_{\mathbf{r}}$ :

$$
g_{\mathbf{r}}(\mathbf{w}, t)=g(\mathbf{w}, 0)+\frac{1}{|\mathbf{r}|} \int_{0}^{t} Q\left(g_{\mathbf{r}}, g_{\mathbf{r}}\right)(\mathbf{w}, \tau) \mathrm{d} \tau, \quad \forall t \geq 0 .
$$

Multiplying the equation by $w_{i}$ and integrating with respect to $\mathbf{w}$ on $\mathbb{R}^{m}$, by (2.6) 
and (2.28) we compute

$$
\begin{aligned}
&\left|\int_{\mathbb{R}^{m}} w_{i} g_{\mathbf{r}}(\mathbf{w}, t) \mathrm{d} \mathbf{w}-\int_{\mathbb{R}^{m}} w_{i} g(\mathbf{w}, 0) \mathrm{d} \mathbf{w}\right| \\
&= \frac{1}{|\mathbf{r}|} \mid \int_{0}^{t} \int_{\mathbb{R}^{m} \times \mathbb{R}^{m}} \int_{\mathbb{R}_{+}^{m} \times \mathbb{R}_{+}^{m}} \rho(\mathbf{x}) \rho\left(\mathbf{x}_{*}\right) g_{\mathbf{r}}(\mathbf{w}, \tau) g_{\mathbf{r}}\left(\mathbf{w}_{*}, \tau\right) \\
&\left(1-\prod_{j=1}^{m} \mathbb{1}_{\left\{\mathbf{w}_{j}^{\prime} \geq 0\right\}} \mathbb{1}_{\left\{\mathbf{w}_{j *}^{\prime} \geq 0\right\}}\right)\left(-x_{i} w_{i}\right) \mathrm{d} \mathbf{w} \mathrm{d} \mathbf{w}_{*} \mathrm{~d} \mathbf{x} \mathrm{d} \mathbf{x}_{*} \mathrm{~d} \tau \mid \\
& \leq \frac{1}{|\mathbf{r}|} \int_{0}^{t} \mathrm{~d} \tau \int_{\mathbb{R}_{+}^{m} \times \mathbb{R}_{+}^{m}} w_{i} g_{\mathbf{r}}(\mathbf{w}, \tau) g_{\mathbf{r}}\left(\mathbf{w}_{*}, \tau\right) \mathrm{d} \mathbf{w} \mathbf{d}_{*} \int_{\mathbb{R}^{m} \times \mathbb{R}^{m}} \rho(\mathbf{x}) \\
& \times \rho\left(\mathbf{x}_{*}\right)\left|x_{i}\right|\left[\sum_{j=1}^{m} \mathbb{1}_{\left\{x_{j}<r_{j}-1\right\}}+\sum_{j=1}^{m} \mathbb{1}_{\left.\left\{x_{j *}<r_{j}-1\right\}\right]} \mathrm{d} \mathbf{x} \mathrm{d} \mathbf{x}_{*} .\right.
\end{aligned}
$$

Taking $\sigma_{i}^{2}=\lambda_{i} r_{i}$ and using the Hölder inequality, under the assumption (2.5) we obtain

$$
\begin{aligned}
& \frac{1}{|\mathbf{r}|} \int_{0}^{t} \mathrm{~d} \tau \int_{\mathbb{R}_{+}^{m} \times \mathbb{R}_{+}^{m}} w_{i} g_{\mathbf{r}}(\mathbf{w}, \tau) g_{\mathbf{r}}\left(\mathbf{w}_{*}, \tau\right) \mathrm{d} \mathbf{w} \mathrm{d} \mathbf{w}_{*} \int_{\mathbb{R}^{m} \times \mathbb{R}^{m}}\left|x_{i}\right| \mathbb{1}_{\left\{x_{j}<r_{j}-1\right\}} \rho(\mathbf{x}) \rho\left(\mathbf{x}_{*}\right) \mathrm{d} \mathbf{x} \mathrm{d} \mathbf{x}_{*} \\
\leq & \frac{1}{|\mathbf{r}|\left(1-r_{j}\right)} \int_{0}^{t} \mathrm{~d} \tau \int_{\mathbb{R}_{+}^{m}} w_{i} g_{\mathbf{r}}(\mathbf{w}, \tau) \mathrm{d} \mathbf{w} \int_{\mathbb{R}^{m}}\left|x_{i}\right|\left|x_{j}\right| \mathbb{1}_{\left\{x_{j}<r_{j}-1\right\}} \rho(\mathbf{x}) \mathrm{d} \mathbf{x} \leq L_{j}
\end{aligned}
$$

where

$$
L_{j}:=2 \sqrt{\lambda_{i} \lambda_{j}} \int_{0}^{t}\left(\int_{\mathbb{R}^{m}}|\mathbf{w}|^{2} g_{\mathbf{r}}(\mathbf{w}, \tau) \mathrm{d} \mathbf{w}\right)^{\frac{1}{2}} \int_{\mathbb{R}^{m-1} \times\left\{y_{j} \in \mathbb{R}^{1} \mid y_{j}<\frac{r_{j}-1}{\sigma_{j}}\right\}}\left|y_{i}\right|\left|y_{j}\right| \rho_{0}(\mathbf{y}) \mathrm{d} \mathbf{y d} \tau .
$$

Note that $\int_{\mathbb{R}^{m}} x_{i}^{2} \rho_{0}(\mathbf{x}) \mathrm{d} \mathbf{x}=1$ by the assumption (2.6). Thus $L_{j} \rightarrow 0$ as $|\mathbf{r}| \rightarrow 0^{+}$. In addition, using the Hölder inequality again, we obtain

$$
\begin{gathered}
\frac{1}{|\mathbf{r}|} \int_{0}^{t} \mathrm{~d} \tau \int_{\mathbb{R}_{+}^{m} \times \mathbb{R}_{+}^{m}} w_{i} g_{\mathbf{r}}(\mathbf{w}, \tau) g_{\mathbf{r}}\left(\mathbf{w}_{*}, \tau\right) \mathrm{d} \mathbf{w} \mathrm{d} \mathbf{w}_{*} \int_{\mathbb{R}^{m} \times \mathbb{R}^{m}} \mathbb{1}_{\left\{x_{j *}<r_{j}-1\right\}} \\
\times\left|x_{i}\right| \rho(\mathbf{x}) \rho\left(\mathbf{x}_{*}\right) \mathrm{d} \mathbf{x} \mathrm{d} \mathbf{x}_{*} \leq L_{j *}
\end{gathered}
$$

where

$$
L_{j *}:=4 \sigma_{i} \lambda_{j} \int_{0}^{t}\left(\int_{\mathbb{R}^{m}}|\mathbf{w}|^{2} g_{\mathbf{r}}(\mathbf{w}, \tau) \mathrm{d} \mathbf{w}\right)^{\frac{1}{2}} \mathrm{~d} \tau
$$

These estimates together with (3.1) prove the lemma.

In order to prove the $L^{1}$-weak compactness of $\left\{g_{\mathbf{r}}(\cdot, t)\right\}_{\mathbf{r}, t}$ in the next section, we need an $L^{2}$-estimate of $g_{\mathbf{r}}(\cdot, t)$, which we prove in the following lemma.

LEMma 3.3. Suppose $0 \leq g(\cdot, 0) \in L^{2}\left(\mathbb{R}^{m}\right) \cap L^{1}\left(\mathbb{R}^{m}\right)$ and $E\left(\left|\zeta_{i}\right|^{2 m+2}\right)<\infty(1 \leq i \leq m)$. Let $|\mathbf{r}|$ be given in (2.31). Assume that

$$
\lim _{|\mathbf{r}| \rightarrow 0^{+}} \frac{r_{i}}{|\mathbf{r}|}:=q_{i}>0, \quad 1 \leq i \leq m
$$


and (2.5)-(2.6) hold. Then for all $0<|\mathbf{r}| \leq \frac{1}{2}$,

$$
\int_{\mathbb{R}^{m}} g_{\mathbf{r}}^{2}(\mathbf{w}, t) \mathrm{d} \mathbf{w} \leq e^{a t} \int_{\mathbb{R}^{m}} g^{2}(\mathbf{w}, 0) \mathrm{d} \mathbf{w}, \quad t \geq 0
$$

where the constant a depends only on $\lambda_{i}, E\left(\left|\zeta_{i}\right|^{2 m+2}\right)$, and the dimension $m$.

Proof. By Theorem 2.6, the equation

$$
\begin{aligned}
\int_{\mathbb{R}^{m}} g_{\mathbf{r}}^{2}(\mathbf{w}, t) \mathrm{d} \mathbf{w}= & \int_{\mathbb{R}^{m}} g^{2}(\mathbf{w}, 0) \mathrm{d} \mathbf{w}+\frac{2}{|\mathbf{r}|} \int_{0}^{t} \int_{\mathbb{R}^{m} \times \mathbb{R}^{m}} \int_{\mathbb{R}^{m} \times \mathbb{R}^{m}} \rho(\mathbf{x}) \rho\left(\mathbf{x}_{*}\right) \\
& \times\left(\prod_{i=1}^{m} \mathbb{1}_{\left\{w_{i} \geq 0\right\}} \mathbb{1}_{\left\{w_{i *} \geq 0\right\}} \mathbb{1}_{\left\{w_{i}^{\prime} \geq 0\right\}} \mathbb{1}_{\left\{w_{i *}^{\prime} \geq 0\right\}}\right)\left(g_{\mathbf{r}}(\mathbf{w}, \tau) g_{\mathbf{r}}\left(\mathbf{w}_{*}, \tau\right)\right. \\
& \left.\times g_{\mathbf{r}}\left(\mathbf{w}^{\prime}, \tau\right)-g_{\mathbf{r}}^{2}(\mathbf{w}, \tau) g_{\mathbf{r}}\left(\mathbf{w}_{*}, \tau\right)\right) \mathrm{d} \mathbf{x} \mathrm{d} \mathbf{x}_{*} \mathrm{~d} \mathbf{w}_{*} \mathrm{~d} \mathbf{w} \mathrm{d} \tau
\end{aligned}
$$

holds for every $0<|\mathbf{r}| \leq \frac{1}{2}$. Define a decomposition $\mathbb{R}^{2 m}=\Omega_{1} \bigcup \Omega_{2}$ with $\Omega_{1}=\left\{\left(\mathbf{x}, \mathbf{x}_{*}\right) \in\right.$ $\left.\mathbb{R}^{2 m}|| x_{i}\left|\leq\left(1-r_{i}\right) / 2,\right| x_{i *} \mid \leq\left(1-r_{i}\right) / 2,1 \leq i \leq m\right\}$ and $\Omega_{2}=\mathbb{R}^{2 m} \backslash \Omega_{1}$. Since

$$
g_{\mathbf{r}}(\mathbf{w}, \tau) g_{\mathbf{r}}\left(\mathbf{w}^{\prime}, \tau\right) \leq \frac{1}{2}\left(g_{\mathbf{r}}^{2}(\mathbf{w}, \tau)+g_{\mathbf{r}}^{2}\left(\mathbf{w}^{\prime}, \tau\right)\right)
$$

and

$$
g_{\mathbf{r}}\left(\mathbf{w}_{*}, \tau\right) g_{\mathbf{r}}\left(\mathbf{w}^{\prime}, \tau\right) \leq \frac{1}{2}\left(g_{\mathbf{r}}^{2}\left(\mathbf{w}_{*}, \tau\right)+g_{\mathbf{r}}^{2}\left(\mathbf{w}^{\prime}, \tau\right)\right),
$$

it follows that

$$
\begin{gathered}
\frac{2}{|\mathbf{r}|} \int_{0}^{t} \int_{\mathbb{R}^{m} \times \mathbb{R}^{m}} \int_{\mathbb{R}^{m} \times \mathbb{R}^{m}} \rho(\mathbf{x}) \rho\left(\mathbf{x}_{*}\right)\left(\prod_{i=1}^{m} \mathbb{1}_{\left\{w_{i} \geq 0\right\}} \mathbb{1}_{\left\{w_{i *} \geq 0\right\}} \mathbb{1}_{\left\{w_{i}^{\prime} \geq 0\right\}} \mathbb{1}_{\left\{w_{i *}^{\prime} \geq 0\right\}}\right) \\
\times\left(g_{\mathbf{r}}(\mathbf{w}, \tau) g_{\mathbf{r}}\left(\mathbf{w}_{*}, \tau\right) g_{\mathbf{r}}\left(\mathbf{w}^{\prime}, \tau\right)-g_{\mathbf{r}}^{2}(\mathbf{w}, \tau) g_{\mathbf{r}}\left(\mathbf{w}_{*}, \tau\right)\right) \mathrm{d} \mathbf{x} \mathrm{d} \mathbf{x}_{*} \mathrm{~d} \mathbf{w}_{*} \mathrm{~d} \mathbf{w} \mathrm{d} \tau \\
\leq \frac{1}{|\mathbf{r}|} \int_{0}^{t} \int_{\mathbb{R}^{m} \times \mathbb{R}^{m}} \int_{\Omega_{1}} \rho(\mathbf{x}) \rho\left(\mathbf{x}_{*}\right)\left(\prod_{i=1}^{m} \mathbb{1}_{\left\{w_{i} \geq 0\right\}} \mathbb{1}_{\left\{w_{i *} \geq 0\right\}} \mathbb{1}_{\left\{w_{i}^{\prime} \geq 0\right\}} \mathbb{1}_{\left\{w_{i *}^{\prime} \geq 0\right\}}\right) \\
\quad \times\left(g_{\mathbf{r}}\left(\mathbf{w}_{*}, \tau\right) g_{\mathbf{r}}^{2}\left(\mathbf{w}^{\prime}, \tau\right)-g_{\mathbf{r}}\left(\mathbf{w}_{*}, \tau\right) g_{\mathbf{r}}^{2}(\mathbf{w}, \tau)\right) \mathrm{d} \mathbf{x} \mathrm{d} \mathbf{x}_{*} \mathrm{~d} \mathbf{w}_{*} \mathrm{~d} \mathbf{w d} \tau \\
+\frac{1}{|\mathbf{r}|} \int_{0}^{t} \int_{\mathbb{R}^{m} \times \mathbb{R}^{m}} \int_{\Omega_{2}} \rho(\mathbf{x}) \rho\left(\mathbf{x}_{*}\right)\left(\prod_{i=1}^{m} \mathbb{1}_{\left\{w_{i} \geq 0\right\}} \mathbb{1}_{\left\{w_{i *} \geq 0\right\}} \mathbb{1}_{\left\{w_{i}^{\prime} \geq 0\right\}} \mathbb{1}_{\left\{w_{i *}^{\prime} \geq 0\right\}}\right) \\
\quad \times g_{\mathbf{r}}(\mathbf{w}, \tau)\left(g_{\mathbf{r}}^{2}\left(\mathbf{w}_{*}, \tau\right)+g_{\mathbf{r}}^{2}\left(\mathbf{w}^{\prime}, \tau\right)\right) \mathrm{d} \mathbf{x} \mathrm{d} \mathbf{x}_{*} \mathrm{~d} \mathbf{w}_{*} \mathrm{~d} \mathbf{w} \mathrm{d} \tau \\
=: I_{1}+I_{2} . \quad
\end{gathered}
$$

Notice that for any $\mathbf{x}, \mathbf{x}_{*} \in \Omega_{1}$, if $\mathbf{w}, \mathbf{w}_{*} \in \mathbb{R}_{+}^{m}$ then $\mathbf{w}^{\prime}, \mathbf{w}_{*}^{\prime} \in \mathbb{R}_{+}^{m}$. Thus, by a change of variables (since $1-r_{i}+x_{i} \geq \frac{1-r_{i}}{2}$ when $\left|x_{i}\right| \leq \frac{1-r_{i}}{2}$ ), we have

$$
\begin{aligned}
& I_{1} \leq \frac{1}{|\mathbf{r}|} \int_{0}^{t} \mathrm{~d} \tau \int_{\Omega_{1}} \frac{1}{\prod_{i=1}^{m}\left(1-r_{i}+x_{i}\right)} \rho(\mathbf{x}) \rho\left(\mathbf{x}_{*}\right) \mathrm{d} \mathbf{x} \mathrm{d} \mathbf{x}_{*} \int_{\mathbb{R}_{+}^{m} \times \mathbb{R}_{+}^{m}} g_{\mathbf{r}}\left(\mathbf{w}_{*}, \tau\right) g_{\mathbf{r}}^{2}(\mathbf{w}, \tau) \mathrm{d} \mathbf{w}_{*} \mathrm{~d} \mathbf{w} \\
& -\frac{1}{|\mathbf{r}|} \int_{0}^{t} \mathrm{~d} \tau \int_{\Omega_{1}} \rho(\mathbf{x}) \rho\left(\mathbf{x}_{*}\right) \mathrm{d} \mathbf{x} \mathrm{d} \mathbf{x}_{*} \int_{\mathbb{R}_{+}^{m} \times \mathbb{R}_{+}^{m}} g_{\mathbf{r}}^{2}(\mathbf{w}, \tau) g_{\mathbf{r}}\left(\mathbf{w}_{*}, \tau\right) \mathrm{d} \mathbf{w}_{*} \mathrm{~d} \mathbf{w} \\
& \leq \frac{1}{|\mathbf{r}|} \int_{0}^{t} \mathrm{~d} \tau \int_{\mathbb{R}_{+}^{m}} g_{\mathbf{r}}^{2}(\mathbf{w}, \tau) \mathrm{d} \mathbf{w} \int_{\Omega_{1}}\left(\frac{1}{\prod_{i=1}^{m}\left(1-r_{i}+x_{i}\right)}-1\right) \rho(\mathbf{x}) \rho\left(\mathbf{x}_{*}\right) \mathrm{d} \mathbf{x} \mathrm{d} \mathbf{x}_{*} .
\end{aligned}
$$


Using (2.6) and the identity

$$
\prod_{i=1}^{m}\left(1+a_{i}\right)=1+\sum_{i=1}^{m} a_{i}+\sum_{1 \leq i_{1} \leq i_{2} \leq m} a_{i_{1}} a_{i_{2}}+\sum_{1 \leq i_{1} \leq i_{2} \leq i_{3} \leq m} a_{i_{1}} a_{i_{2}} a_{i_{3}}+\cdots+\prod_{i=1}^{m} a_{i},
$$

we compute

$$
\begin{aligned}
& \frac{1}{|\mathbf{r}|} \int_{\Omega_{1}}\left(\frac{1}{\prod_{i=1}^{m}\left(1-r_{i}+x_{i}\right)}-1\right) \rho(\mathbf{x}) \rho\left(\mathbf{x}_{*}\right) \mathrm{d} \mathbf{x} \mathrm{d} \mathbf{x}_{*} \\
= & \frac{1}{2|\mathbf{r}|} \int_{\Omega_{1}}\left(\frac{1}{\prod_{i=1}^{m}\left(1-r_{i}+x_{i}\right)}+\frac{1}{\prod_{i=1}^{m}\left(1-r_{i}-x_{i}\right)}-2\right) \rho(\mathbf{x}) \rho\left(\mathbf{x}_{*}\right) \mathrm{d} \mathbf{x} \mathrm{d} \mathbf{x}_{*} \\
\leq & \frac{8^{m}}{2 \cdot 3^{m}|\mathbf{r}|} \int_{\Omega_{1}}\left[\prod_{i=1}^{m}\left(1-\frac{x_{i}}{1-r_{i}}\right)+\prod_{i=1}^{m}\left(1+\frac{x_{i}}{1-r_{i}}\right)-2 \prod_{i=1}^{m}\left(1-r_{i}-\frac{x_{i}^{2}}{1-r_{i}}\right)\right] \rho(\mathbf{x}) \rho\left(\mathbf{x}_{*}\right) \mathrm{d} \mathbf{x} \mathrm{d} \mathbf{x}_{*} \\
\leq & c(m) \sum_{i=1}^{m}\left(4 \lambda_{i}+1\right) .
\end{aligned}
$$

Substituting this into (3.13), we obtain

$$
I_{1} \leq c(m) \sum_{i=1}^{m}\left(4 \lambda_{i}+1\right) \int_{0}^{t} \mathrm{~d} \tau \int_{\mathbb{R}^{m}} g_{\mathbf{r}}^{2}(\mathbf{w}, \tau) \mathrm{d} \mathbf{w} .
$$

On the other hand, for every element in $\Omega_{2}$ there exists at least one component, denoted by $x_{i_{0}}$ ( or $x_{i_{0} *}$ ), such that $\left|x_{i_{0}}\right|>\frac{1-r_{i_{0}}}{2}$ (or $\left|x_{i_{0} *}\right|>\frac{1-r_{i_{0}}}{2}$ ). We only estimate $I_{2}$ for the case where $\left|x_{i_{0}}\right|>\frac{1-r_{i_{0}}}{2}$. The other case is exactly the same because of the same distribution of $\boldsymbol{\eta}$ and $\boldsymbol{\eta}_{*}$. Moreover, we suppose $|\mathbf{r}| / r_{k} \leq c\left(q_{k}\right)$ for each $k$ since $r_{k} /|\mathbf{r}| \rightarrow q_{k}(>0)$ is considered. Here $c\left(q_{k}\right)$ is a constant depending only on $q_{k}$. Taking $\sigma_{i}^{2}=\lambda_{i} r_{i}$, we obtain an estimate by an analysis similar to that in (2.29):

$$
\begin{aligned}
& \frac{1}{|\mathbf{r}|} \int_{0}^{t} \mathrm{~d} \tau \int_{\mathbb{R}^{m}} g_{\mathbf{r}}^{2}\left(\mathbf{w}_{*}, \tau\right) \mathrm{d} \mathbf{w}_{*} \int_{\Omega_{2}} \rho(\mathbf{x}) \mathrm{d} \mathbf{x} \\
& +\frac{1}{|\mathbf{r}| \prod_{k=1}^{m} r_{k}} \int_{0}^{t} \mathrm{~d} \tau \int_{\mathbb{R}^{m}} g_{\mathbf{r}}^{2}\left(\mathbf{w}_{*}, \tau\right) \mathrm{d} \mathbf{w}_{*} \int_{\Omega_{2}} \rho(\mathbf{x}) \mathrm{d} \mathbf{x} \\
\leq & {\left[\lambda_{i_{0}}+\lambda_{i_{0}}^{m+1} E\left(\left|\zeta_{i_{0}}\right|^{2 m+2}\right) \prod_{k=1}^{m} c\left(q_{k}\right)\right] \int_{0}^{t} \mathrm{~d} \tau \int_{\mathbb{R}^{m}} g_{\mathbf{r}}^{2}(\mathbf{w}, \tau) \mathrm{d} \mathbf{w}, }
\end{aligned}
$$

which gives

$$
I_{2} \leq\left[\sum_{i=1}^{m} \lambda_{i}+\sum_{i=1}^{m} \lambda_{i}^{m+1} E\left(\left|\zeta_{i}\right|^{2 m+2}\right) \prod_{k=1}^{m} c\left(q_{k}\right)\right] \int_{0}^{t} \mathrm{~d} \tau \int_{\mathbb{R}^{m}} g_{\mathbf{r}}^{2}(\mathbf{w}, \tau) \mathrm{d} \mathbf{w} .
$$

Finally, combining the estimates for $I_{1}, I_{2}$ with (3.11)-(3.12) and using the Gronwall inequality, we prove (3.10) with $a=c(m)\left(\sum_{i=1}^{m} 4 \lambda_{i}+1\right)+\sum_{i=1}^{m} \lambda_{i}+$ $\sum_{i=1}^{m} \lambda_{i}^{m+1} E\left(\left|\zeta_{i}\right|^{2 m+2}\right) \prod_{k=1}^{m} c\left(q_{k}\right)$. 


\section{Asymptotic limit of the multi-dimensional model}

Throughout this section, we always assume that (2.5)-(2.6), (3.9) hold and $E\left(\left|\zeta_{i}\right|^{2 m+2}\right)<\infty(1 \leq i \leq m)$. Here recall that $|\mathbf{r}|=\max _{1 \leq i \leq 1}\left\{r_{i}\right\}$.

Theorem 4.1. Assume that $0 \leq g(\cdot, 0) \in L^{2}\left(\mathbb{R}^{m}\right) \cap L_{2}^{1}\left(\mathbb{R}^{m}\right)$ and that $\lambda_{i}<\infty(1 \leq i \leq$ $m)$ are positive constants such that $\sigma_{i}^{2}=\lambda_{i} r_{i}$. Let $\left\{g_{\mathbf{r}}(\mathbf{w}, t)\right\}_{\mathbf{r}}$ be a family of timescaled mild solutions of (2.8) with the same initial datum $g(\mathbf{w}, 0)=f(\mathbf{w}, 0)$. Then $\forall\left|\mathbf{r}_{n}\right| \rightarrow 0^{+}(n \rightarrow \infty)$, there exists a subsequence $\left\{g_{\mathbf{r}_{n_{k}}}(\mathbf{w}, t)\right\}_{k=1}^{\infty}$ of $\left\{g_{\mathbf{r}_{n}}(\mathbf{w}, t)\right\}_{n=1}^{\infty}$ and a function $0 \leq g(\mathbf{w}, t) \in C\left([0, \infty), L^{1}\left(\mathbf{R}_{+}^{m}\right)\right)$, such that $g_{\mathbf{r}_{n_{k}}}(\mathbf{w}, t)$ converges weakly to $g(\mathbf{w}, t)$ as $k \rightarrow \infty$. Moreover, $g(\mathbf{w}, t)$ is a weak solution of the following FokkerPlanck equation with the initial datum $g(\mathbf{w}, 0)$ :

$\frac{\partial g(\mathbf{w}, t)}{\partial t}=\sum_{i=1}^{m} q_{i} \frac{\partial}{\partial w_{i}}\left[\left(w_{i}-S_{i}\right) g(\mathbf{w}, t)\right]+\frac{1}{2} \sum_{i=1}^{m} \sum_{j=1}^{m} \kappa_{i j} \sqrt{q_{i} q_{j} \lambda_{i} \lambda_{j}} \frac{\partial^{2}}{\partial w_{i} \partial w_{j}}\left[w_{i} w_{j} g(\mathbf{w}, t)\right]$,

where $q_{i}>0$ is given by (3.9), $S_{i}=\int_{\mathbb{R}^{m}} w_{i} g(\mathbf{w}, 0) \mathrm{d} \mathbf{w}$ is the initial amount of money invested in asset $i$, and $\kappa_{i j}$ is the correlation coefficient between the rate of return for asset $i$ and asset $j$.

REMARK 4.2. For two assets, $i$ and $j$, recall that the covariance of the rates of return is defined as: $\sigma_{i j}=E\left\{\left[\eta_{i}-E\left(\eta_{i}\right)\right]\left[\eta_{j}-E\left(\eta_{j}\right)\right]\right\}$, and that the correlation coefficient is obtained by standardizing the covariance: $\kappa_{i j}=\frac{\sigma_{i j}}{\sigma_{i} \sigma_{j}}$.

REMARK 4.3. The assumption of $g(\cdot, 0) \in L^{2}\left(\mathbb{R}^{m}\right)$ (together with the assumption that $\left.E\left(\left|\zeta_{i}\right|^{2 m+2}\right)<\infty\right)$ is only used to prove the $L^{1}$-weak compactness of $\left\{g_{\mathbf{r}}(\mathbf{w}, t)\right\}_{\mathbf{r}, t}$. Even though the condition of $g(\cdot, 0) \in L^{2}\left(\mathbb{R}^{m}\right)$ is very strong, it can provide some symmetries to get the cancellation property in the estimate for $I_{1}$, as one can see in (3.13). Of course if one only wants to get the limit equation in a distributional sense, the assumption of $g(\cdot, 0) \in L^{2}\left(\mathbb{R}^{m}\right) \cap L_{2}^{1}\left(\mathbb{R}^{m}\right)$ can be weakened to that of $g(\cdot, 0) \in L_{2}^{1}\left(\mathbb{R}^{m}\right)$.

\section{Proof of the Theorem 4.1.}

Proof. Let $\varphi(\mathbf{w})$ be a test function which belongs to $C_{c}^{\infty}\left(\mathbb{R}_{>0}^{m}\right)$. Then the equation

$$
\begin{aligned}
& \int_{\mathbb{R}^{m}} \varphi(\mathbf{w}) g_{\mathbf{r}}(\mathbf{w}, t) \mathrm{d} \mathbf{w} \\
= & \int_{\mathbb{R}^{m}} \varphi(\mathbf{w}) g(\mathbf{w}, 0) \mathrm{d} \mathbf{w}-\frac{1}{|\mathbf{r}|} \int_{0}^{t} \int_{\mathbb{R}^{m} \times \mathbb{R}^{m}} \int_{\mathbb{R}^{m} \times \mathbb{R}^{m}} \rho(\mathbf{x}) \\
& \times \rho\left(\mathbf{x}_{*}\right)\left(\prod_{i=1}^{m} \mathbb{1}_{\left\{w_{i} \geq 0\right\}} \mathbb{1}_{\left\{w_{i *} \geq 0\right\}}\right)\left[1-\left(\prod_{i=1}^{m} \mathbb{1}_{\left\{w_{i}^{\prime} \geq 0\right\}} \mathbb{1}_{\left\{w_{i *}^{\prime} \geq 0\right\}}\right)\right] g_{\mathbf{r}}(\mathbf{w}, \tau) g_{\mathbf{r}}\left(\mathbf{w}_{*}, \tau\right) \\
& \times\left[\varphi\left(\mathbf{w}^{\prime}\right)-\varphi(\mathbf{w})\right] \mathrm{d} \mathbf{x} \mathrm{d} \mathbf{x}_{*} \mathrm{~d} \mathbf{w} \mathrm{d} \mathbf{w}_{*} \mathrm{~d} \tau+\frac{1}{|\mathbf{r}|} \int_{0}^{t} \int_{\mathbb{R}^{m} \times \mathbb{R}^{m}} \int_{\mathbb{R}^{m} \times \mathbb{R}^{m}} \rho(\mathbf{x}) \rho\left(\mathbf{x}_{*}\right) \\
& \times\left(\prod_{i=1}^{m} \mathbb{1}_{\left\{w_{i} \geq 0\right\}} \mathbb{1}_{\left\{w_{i *} \geq 0\right\}}\right)\left[\varphi\left(\mathbf{w}^{\prime}\right)-\varphi(\mathbf{w})\right] g_{\mathbf{r}}(\mathbf{w}, \tau) g_{\mathbf{r}}\left(\mathbf{w}_{*}, \tau\right) \mathrm{d} \mathbf{x} \mathrm{d} \mathbf{x}_{*} \mathrm{~d} \mathbf{w} \mathrm{d} \mathbf{w}_{*} \mathrm{~d} \tau
\end{aligned}
$$


holds by (3.3). Recall Taylor's formula

$$
\begin{aligned}
\varphi\left(\mathbf{w}^{\prime}\right)-\varphi(\mathbf{w})= & \left\langle\nabla \varphi, \mathbf{w}^{\prime}-\mathbf{w}\right\rangle+\frac{1}{2} \sum_{i=1}^{m} \sum_{j=1}^{m}\left(w_{i}^{\prime}-w_{i}\right)\left(w_{j}^{\prime}-w_{j}\right) \frac{\partial^{2} \varphi(\mathbf{w})}{\partial w_{i} \partial w_{j}} \\
& +\frac{1}{2} \sum_{i=1}^{m} \sum_{j=1}^{m}\left(w_{i}^{\prime}-w_{i}\right)\left(w_{j}^{\prime}-w_{j}\right)\left(\frac{\partial^{2} \varphi(\tilde{\mathbf{w}})}{\partial w_{i} \partial w_{j}}-\frac{\partial^{2} \varphi(\mathbf{w})}{\partial w_{i} \partial w_{j}}\right),
\end{aligned}
$$

where $\tilde{\mathbf{w}}=\mathbf{w}+\theta\left(\mathbf{w}^{\prime}-\mathbf{w}\right), \theta \in(0,1)$. Inserting this expansion into (4.2), the Equation (4.2) becomes

$$
\begin{aligned}
& \int_{\mathbb{R}^{m}} \varphi(\mathbf{w}) g_{\mathbf{r}}(\mathbf{w}, t) \mathrm{d} \mathbf{w} \\
= & \int_{\mathbb{R}^{m}} \varphi(\mathbf{w}) g(\mathbf{w}, 0) \mathrm{d} \mathbf{w}+\frac{1}{|\mathbf{r}|} \int_{0}^{t} \int_{\mathbb{R}^{m} \times \mathbb{R}^{m}} \int_{\mathbb{R}^{m} \times \mathbb{R}^{m}} \rho(\mathbf{x}) \rho\left(\mathbf{x}_{*}\right) \\
& \times g_{\mathbf{r}}(\mathbf{w}, \tau) g_{\mathbf{r}}\left(\mathbf{w}_{*}, \tau\right)\left(\prod_{i=1}^{m} \mathbb{1}_{\left\{w_{i} \geq 0\right\}} \mathbb{1}_{\left\{w_{i *} \geq 0\right\}}\right)\left[\left\langle\nabla \varphi, \mathbf{w}^{\prime}-\mathbf{w}\right\rangle\right. \\
& \left.+\frac{1}{2} \sum_{i=1}^{m} \sum_{j=1}^{m}\left(w_{i}^{\prime}-w_{i}\right)\left(w_{j}^{\prime}-w_{j}\right) \frac{\partial^{2} \varphi(\mathbf{w})}{\partial w_{i} \partial w_{j}}\right] \mathrm{d} \mathbf{x} \mathrm{d} \mathbf{x}_{*} \mathrm{~d} \mathbf{w} \mathrm{d} \mathbf{w}_{*} \mathrm{~d} \tau+\mathcal{R}_{1}(\mathbf{r})+\mathcal{R}_{2}(\mathbf{r}),
\end{aligned}
$$

where

$$
\begin{aligned}
\mathcal{R}_{1}(\mathbf{r})= & \frac{1}{|\mathbf{r}|} \int_{0}^{t} \int_{\mathbb{R}^{m} \times \mathbb{R}^{m}} \int_{\mathbb{R}^{m} \times \mathbb{R}^{m}} \rho(\mathbf{x}) \rho\left(\mathbf{x}_{*}\right)\left(\prod_{i=1}^{m} \mathbb{1}_{\left\{w_{i} \geq 0\right\}} \mathbb{1}_{\left\{w_{i *} \geq 0\right\}}\right) g_{\mathbf{r}}(\mathbf{w}, \tau) g_{\mathbf{r}}\left(\mathbf{w}_{*}, \tau\right) \\
& \times \frac{1}{2} \sum_{i=1}^{m} \sum_{j=1}^{m}\left(w_{i}^{\prime}-w_{i}\right)\left(w_{j}^{\prime}-w_{j}\right)\left(\frac{\partial^{2} \varphi(\tilde{\mathbf{w}})}{\partial w_{i} \partial w_{j}}-\frac{\partial^{2} \varphi(\mathbf{w})}{\partial w_{i} \partial w_{j}}\right) \mathrm{d} \mathbf{x} \mathrm{d} \mathbf{x}_{*} \mathrm{~d} \mathbf{w} \mathrm{d} \mathbf{w}_{*} \mathrm{~d} \tau \\
\mathcal{R}_{2}(\mathbf{r})= & -\frac{1}{|\mathbf{r}|} \int_{0}^{t} \int_{\mathbb{R}^{m} \times \mathbb{R}^{m}} \int_{\mathbb{R}^{m} \times \mathbb{R}^{m}} \rho(\mathbf{x}) \rho\left(\mathbf{x}_{*}\right)\left(\prod_{i=1}^{m} \mathbb{1}_{\left\{w_{i} \geq 0\right\}} \mathbb{1}_{\left\{w_{i *} \geq 0\right\}}\right) \\
& \times\left(1-\prod_{i=1}^{m} \mathbb{1}_{\left\{w_{i}^{\prime} \geq 0\right\}} \mathbb{1}_{\left\{w_{i *}^{\prime} \geq 0\right\}}\right)\left[\varphi\left(\mathbf{w}^{\prime}\right)-\varphi(\mathbf{w})\right] \\
& \times g_{\mathbf{r}}(\mathbf{w}, \tau) g_{\mathbf{r}}\left(\mathbf{w}_{*}, \tau\right) \mathrm{d} \mathbf{x} \mathrm{d} \mathbf{x}_{*} \mathrm{~d} \mathbf{w} \mathrm{d} \mathbf{w}_{*} \mathrm{~d} \tau
\end{aligned}
$$

Next, we divide the proof into three steps. Let us estimate successively each of the two terms $\mathcal{R}_{1}(\mathbf{r}), \mathcal{R}_{2}(\mathbf{r})$.

Step 1: Proof of $\mathcal{R}_{1}(\mathbf{r}) \rightarrow 0$ as $|\mathbf{r}| \rightarrow 0^{+}$. Without loss of generality, we may assume that $0<|\mathbf{r}|<1 / 2$. Now we introduce some notation. Since $\varphi$ is compactly supported, there exists $N>0$ such that supp $\varphi \subset[-N, N]^{m}$. We split $\mathbb{R}^{1}$ into two disjoint sets $\mathbb{R}^{1}=K_{0} \cup K_{1}$, where $K_{0}=[-N, N]$ and $K_{1}=\mathbb{R}^{1} \backslash K_{0}$. Then for each $w_{i} \in$ $\mathbb{R}^{1}$, either $w_{i} \in K_{0}$ or $w_{i} \in K_{1}$. This decomposition of $\mathbb{R}^{1}$ leads to a natural partition of $\mathbb{R}^{m}$

$$
\mathbb{R}^{m}=\left(K_{0} \cup K_{1}\right)^{m}=\bigcup_{\mu \in \Gamma} Q_{\mu}, \quad Q_{\mu}:=K_{\mu(1)} \times K_{\mu(2)} \times \cdots K_{\mu(m)},
$$

where $\Gamma=\{\mu \mid \mu:\{1,2, \ldots, m\} \rightarrow\{0,1\}\}$. For each $\left(\mathbf{x}, \mathbf{w}_{*}\right)$, define

$$
Q_{\mu}^{\left(\mathbf{x}, \mathbf{w}_{*}\right)}=K_{\mu(1)}^{\left(\mathbf{x}, \mathbf{w}_{*}\right)} \times K_{\mu(2)}^{\left(\mathbf{x}, \mathbf{w}_{*}\right)} \times \cdots \times K_{\mu(m)}^{\left(\mathbf{x}, \mathbf{w}_{*}\right)}, \quad \mu \in \Gamma,
$$


with

$$
K_{\mu(i)}^{\left(\mathbf{x}, \mathbf{w}_{*}\right)}= \begin{cases}K_{0}, & \text { if } \mu(i)=0 \\ \left\{w_{i} \in K_{1}|| w_{i}\left|\leq 2 N+2 r_{i}\right| w_{i *}|+2| x_{i}|| w_{i} \mid\right\}, & \text { if } \mu(i)=1\end{cases}
$$

For any $i, j$, let

$$
\Gamma_{i j}=\{\mu \in \Gamma \mid \mu(i)=0 \& \mu(j)=0\}, \quad \Gamma_{i}^{j}=\{\mu \in \Gamma \mid \mu(i)=0 \& \mu(j)=1\} .
$$

Since supp $\varphi \subset[-N, N]^{m}$, we see for each $\left(\mathbf{x}, \mathbf{w}_{*}\right)$ that

$$
\left|\frac{\partial^{2} \varphi(\tilde{\mathbf{w}})}{\partial w_{k} w_{l}}-\frac{\partial^{2} \varphi(\mathbf{w})}{\partial w_{k} w_{l}}\right| \mathbb{1}_{Q_{\mu}}(\mathbf{w}) \leq\left|\frac{\partial^{2} \varphi(\tilde{\mathbf{w}})}{\partial w_{k} w_{l}}-\frac{\partial^{2} \varphi(\mathbf{w})}{\partial w_{k} w_{l}}\right| \mathbb{1}_{Q_{\mu}^{\left(\mathbf{x}, \mathbf{w}_{*}\right)}}(\mathbf{w}),
$$

where $\tilde{\mathbf{w}}$ is the vector given in (4.3): $\tilde{\mathbf{w}}=\mathbf{w}+\theta\left(\mathbf{w}^{\prime}-\mathbf{w}\right), \theta \in(0,1)$. Recall that $a \wedge b=$ $\min \{a, b\}$. Then the mean value theorem gives that

$$
\left|\frac{\partial^{2} \varphi(\tilde{\mathbf{w}})}{\partial w_{k} w_{l}}-\frac{\partial^{2} \varphi(\mathbf{w})}{\partial w_{k} w_{l}}\right| \leq 2\|\varphi\|_{3}\left[\left(\sum_{i=1}^{m}\left|w_{i}^{\prime}-w_{i}\right|\right) \wedge 1\right], \quad k, l=1, \cdots, m,
$$

where $\|\varphi\|_{3}:=\sup _{|\boldsymbol{\beta}| \leq 3}\left|D^{\boldsymbol{\beta}} \varphi\right|$ and $\boldsymbol{\beta}=\left(\beta_{1}, \beta_{2}, \cdots, \beta_{m}\right)$ is a multi-index with $|\boldsymbol{\beta}|=\sum_{i=1}^{m} \beta_{i}$. Then by (2.23), (4.5), and (4.9)-(4.10) we have

$$
\begin{aligned}
\left|\mathcal{R}_{1}(\mathbf{r})\right| \leq & \frac{c(m)\|\varphi\|_{3}}{|\mathbf{r}|} \int_{0}^{t} \mathrm{~d} \tau \int_{\mathbb{R}_{+}^{m}} g_{\mathbf{r}}\left(\mathbf{w}_{*}, \tau\right) \mathrm{d} \mathbf{w}_{*} \int_{\mathbb{R}^{m} \times \mathbb{R}^{m}} \rho(\mathbf{x}) \\
& \times \rho\left(\mathbf{x}_{*}\right) \mathrm{d} \mathbf{x} \mathrm{d} \mathbf{x}_{*} \int_{\bigcup_{\mu \in \Gamma} Q_{\mu}^{\left(\mathbf{x}, \mathbf{w}_{*}\right)}} g_{\mathbf{r}}(\mathbf{w}, \tau)\left[2 \sum_{i, j=1}^{m}\left(r_{i}^{3}\left|w_{i}\right|^{3} \wedge r_{j}^{2}\left|w_{j *}\right|^{2}\right)\right. \\
& +2 \sum_{i, j=1}^{m}\left(r_{i}^{3}\left|w_{i}\right|^{3} \wedge r_{j}^{2}\left|w_{j}\right|^{2}\right)+\sum_{i, j=1}^{m}\left(\left|x_{i}\right|^{3}\left|w_{i}\right|^{3} \wedge r_{j}^{2}\left|w_{j *}\right|^{2}\right) \\
& +\sum_{i, j=1}^{m}\left(\left|x_{i}\right|^{3}\left|w_{i}\right|^{3} \wedge r_{j}^{2}\left|w_{j}\right|^{2}\right)+\sum_{i, i=1}^{m}\left(r_{i}^{3}\left|w_{i *}\right|^{3} \wedge\left|x_{j}\right|^{2}\left|w_{j}\right|^{2}\right) \\
& \left.+\sum_{i, j=1}^{m}\left(r_{i}^{3}\left|w_{i}\right|^{3} \wedge\left|x_{j}\right|^{2}\left|w_{j}\right|^{2}\right)+\sum_{i, j=1}^{m}\left(\left|x_{i}\right|^{3}\left|w_{i}\right|^{3} \wedge\left|x_{j}\right|^{2}\left|w_{j}\right|^{2}\right)\right] \mathrm{d} \mathbf{w} \\
= & : J_{1}+J_{2}+J_{3}+J_{4}+J_{5}+J_{6}+J_{7} .
\end{aligned}
$$

As mentioned before, we have

$$
\int_{\mathbb{R}^{m}} g_{\mathbf{r}}(\mathbf{w}, t) \mathrm{d} \mathbf{w}=\int_{\mathbb{R}^{m}} f(\mathbf{w}, 0) \mathrm{d} \mathbf{w} .
$$

This leads to

$$
J_{1}, J_{2}, J_{3}, J_{4} \leq c(m)\|\varphi\|_{3}|\mathbf{r}| \int_{0}^{t} \mathrm{~d} \tau \int_{\mathbb{R}^{m}}|\mathbf{w}|^{2} g_{\mathbf{r}}(\mathbf{w}, \tau) \mathrm{d} \mathbf{w} \rightarrow 0 \quad\left(|\mathbf{r}| \rightarrow 0^{+}\right) .
$$

(i) The estimate for $J_{5}$. For any $i, j$, let

$$
\begin{aligned}
J_{5}^{i, j}= & \frac{c(m)\|\varphi\|_{3}}{|\mathbf{r}|} \int_{0}^{t} \mathrm{~d} \tau \int_{\mathbb{R}^{m} \times \mathbb{R}^{m}} \rho(\mathbf{x}) \rho\left(\mathbf{x}_{*}\right) \mathrm{d} \mathbf{x} \mathrm{d} \mathbf{x}_{*} \int_{\mathbb{R}_{+}^{m}}\left(\prod_{k=1}^{m} \mathbb{1}_{\left\{w_{k *} \geq 0\right\}}\right) \\
& \times g_{\mathbf{r}}\left(\mathbf{w}_{*}, \tau\right) \mathrm{d} \mathbf{w}_{*} \int_{\bigcup_{\mu \in \Gamma} Q_{\mu}^{\left(\mathbf{x}, \mathbf{w}_{*}\right)}}\left(r_{i}^{3}\left|w_{i *}\right|^{3} \wedge\left|x_{j}\right|^{2}\left|w_{j}\right|^{2}\right) g_{\mathbf{r}}(\mathbf{w}, \tau) \mathrm{d} \mathbf{w} .
\end{aligned}
$$


To prove $J_{5} \rightarrow 0$, it suffices to show that $J_{5}^{i, j} \rightarrow 0$ as $|\mathbf{r}| \rightarrow 0^{+}$since $J_{5}$ is a finite sum of $J_{5}^{i, j}$. By (2.6) we have

$$
\begin{gathered}
J_{5}^{i, j} \leq c(m)\|\varphi\|_{3}|\mathbf{r}| \int_{0}^{t} \mathrm{~d} \tau \int_{\mathbb{R}^{m} \times \mathbb{R}^{m}}\left|x_{j}\right|^{\frac{2}{3}} \rho(\mathbf{x}) \rho\left(\mathbf{x}_{*}\right) \mathrm{d} \mathbf{x} \mathrm{d} \mathbf{x}_{*} \int_{\left\{r_{i}^{3}\left|w_{i *}\right|^{3} \leq\left|x_{j}\right|^{2}\left|w_{j}\right|^{2}\right\}}\left|w_{i *}\right|^{2}\left|w_{j}\right|^{\frac{2}{3}} \\
\times g_{\mathbf{r}}(\mathbf{w}, \tau) g_{\mathbf{r}}\left(\mathbf{w}_{*}, \tau\right) \mathrm{d} \mathbf{w} \mathrm{d} \mathbf{w}_{*}+\frac{c(m)\|\varphi\|_{3}}{|\mathbf{r}|} \int_{0}^{t} \mathrm{~d} \tau \int_{\mathbb{R}^{m} \times \mathbb{R}^{m}}\left|x_{j}\right|^{2} \rho(\mathbf{x}) \\
\times \rho\left(\mathbf{x}_{*}\right) \mathrm{d} \mathbf{x} \mathrm{d} \mathbf{x}_{*} \int_{\left\{r_{i}^{3}\left|w_{i *}\right|^{3}>\left|x_{j}\right|^{2}\left|w_{j}\right|^{2}\right\}} \frac{\left|w_{i *}\right|^{2}}{\left|w_{i *}\right|^{2}}\left|w_{j}\right|^{2} g_{\mathbf{r}}(\mathbf{w}, \tau) g_{\mathbf{r}}\left(\mathbf{w}_{*}, \tau\right) \mathrm{d} \mathbf{w} \mathrm{d} \mathbf{w}_{*} \\
\leq c(m)\|\varphi\|_{3}|\mathbf{r}| \sigma_{j}^{\frac{2}{3}} \int_{0}^{t} \mathrm{~d} \tau \int_{\mathbb{R}^{m} \times \mathbb{R}^{m}}\left|\mathbf{w}_{*}\right|^{2}|\mathbf{w}|^{\frac{2}{3}} g_{\mathbf{r}}(\mathbf{w}, \tau) g_{\mathbf{r}}\left(\mathbf{w}_{*}, \tau\right) \mathrm{d} \mathbf{w} \mathrm{d} \mathbf{w}_{*}
\end{gathered}
$$

From (3.1), we see that $\mathbf{r} \mapsto \int_{\mathbb{R}^{m}}|\mathbf{w}|^{2} g_{\mathbf{r}}(\mathbf{w}, t) \mathrm{d} \mathbf{w}$ is bounded for any $t$. Then, using the Hölder inequality, one sees that the right-hand side of the inequality (4.14) converges to zero as $|\mathbf{r}| \rightarrow 0^{+}$. Thus $J_{5}^{i, j} \rightarrow 0\left(|\mathbf{r}| \rightarrow 0^{+}\right)$, and this proves that $J_{5} \rightarrow 0$.

(ii) The estimate for $J_{6}$. Similarly, let

$$
\begin{aligned}
J_{6}^{i, j}= & \frac{c(m)\|\varphi\|_{3}}{|\mathbf{r}|} \int_{0}^{t} \mathrm{~d} \tau \int_{\mathbb{R}^{m} \times \mathbb{R}^{m}} \rho(\mathbf{x}) \rho\left(\mathbf{x}_{*}\right) \mathrm{d} \mathbf{x} \mathrm{d} \mathbf{x}_{*} \int_{\mathbb{R}_{+}^{m}}\left(\prod_{k=1}^{m} \mathbb{1}_{\left\{w_{k *} \geq 0\right\}}\right) \\
& \times g_{\mathbf{r}}\left(\mathbf{w}_{*}, \tau\right) \mathrm{d} \mathbf{w}_{*} \int_{\bigcup_{\mu \in \Gamma} Q_{\mu}^{\left(\mathbf{x}, \mathbf{w}_{*}\right)}}\left(r_{i}^{3}\left|w_{i}\right|^{3} \wedge\left|x_{j}\right|^{2}\left|w_{j}\right|^{2}\right) g_{\mathbf{r}}(\mathbf{w}, \tau) \mathrm{d} \mathbf{w} .
\end{aligned}
$$

It is enough to prove $J_{6}^{i, j} \rightarrow 0$ as $|\mathbf{r}| \rightarrow 0^{+}$. Split $J_{6}^{i, j}$ into two parts $J_{6,1}^{i, j}+J_{6,2}^{i, j}$,

$$
\begin{aligned}
J_{6,1}^{i, j}= & \frac{c(m)\|\varphi\|_{3}}{|\mathbf{r}|} \int_{0}^{t} \mathrm{~d} \tau \int_{\mathbb{R}^{m} \times \mathbb{R}^{m}} \rho(\mathbf{x}) \rho\left(\mathbf{x}_{*}\right) \mathrm{d} \mathbf{x} \mathrm{d} \mathbf{x}_{*} \int_{\mathbb{R}_{+}^{m}}\left(\prod_{k=1}^{m} \mathbb{1}_{\left\{w_{k *} \geq 0\right\}}\right) \\
& \times g_{\mathbf{r}}\left(\mathbf{w}_{*}, \tau\right) \mathrm{d} \mathbf{w}_{*} \int_{\bigcup_{\mu \in \Gamma_{i}^{j} \cup \Gamma_{i j}} Q_{\mu}^{\left(\mathbf{x}, \mathbf{w}_{*}\right)}}\left(r_{i}^{3}\left|w_{i}\right|^{3} \wedge\left|x_{j}\right|^{2}\left|w_{j}\right|^{2}\right) g_{\mathbf{r}}(\mathbf{w}, \tau) \mathrm{d} \mathbf{w}
\end{aligned}
$$

and

$$
\begin{aligned}
J_{6,2}^{i, j}= & \frac{c(m)\|\varphi\|_{3}}{|\mathbf{r}|} \int_{0}^{t} \mathrm{~d} \tau \int_{\mathbb{R}^{m} \times \mathbb{R}^{m}} \rho(\mathbf{x}) \rho\left(\mathbf{x}_{*}\right) \mathrm{d} \mathbf{x} \mathrm{d} \mathbf{x}_{*} \int_{\mathbb{R}_{+}^{m}}\left(\prod_{k=1}^{m} \mathbb{1}_{\left\{w_{k *} \geq 0\right\}}\right) \\
& \times g_{\mathbf{r}}\left(\mathbf{w}_{*}, \tau\right) \mathrm{d} \mathbf{w}_{*} \int_{\bigcup_{\mu \notin\left(\Gamma_{i}^{j} \cup \Gamma_{i j}\right)}} Q_{\mu}^{\left(\mathbf{x}, \mathbf{w}_{*}\right)} \\
& \left(r_{i}^{3}\left|w_{i}\right|^{3} \wedge\left|x_{j}\right|^{2}\left|w_{j}\right|^{2}\right) g_{\mathbf{r}}(\mathbf{w}, \tau) \mathrm{d} \mathbf{w} .
\end{aligned}
$$

It is easily seen that

$$
J_{6,1}^{i, j} \leq c(m)\|\varphi\|_{3}|\mathbf{r}|^{2} N^{3} t \rightarrow 0 \quad\left(|\mathbf{r}| \rightarrow 0^{+}\right) .
$$


Next we estimate $J_{6,2}^{i, j}$

$$
\begin{aligned}
J_{6,2}^{i, j} \leq & \frac{c(m)\|\varphi\|_{3}}{|\mathbf{r}|} \int_{0}^{t} \mathrm{~d} \tau \int_{\mathbb{R}^{m-2} \times\left\{\left|x_{i}\right| \leq \frac{1}{4},\left|x_{j}\right| \leq \frac{1}{4}\right\}} \rho(\mathbf{x}) \mathrm{d} \mathbf{x} \int_{\mathbb{R}^{m}} g_{\mathbf{r}}\left(\mathbf{w}_{*}, \tau\right) \\
& \times\left[8 r_{i}^{3}\left(4^{3} N^{3}+4^{3} r_{i}^{3}\left|w_{i *}\right|^{3}\right) \wedge 4\left|x_{j}\right|^{2}\left(4^{2} N^{2}+4^{2} r_{j}^{2}\left|w_{j *}\right|^{2}\right)\right] \mathrm{d} \mathbf{w}_{*} \\
& \quad+\frac{c(m)\|\varphi\|_{3}}{|\mathbf{r}|} \int_{0}^{t} \mathrm{~d} \tau \int_{\mathbb{R}^{m-2} \times\left(\mathbb{R}^{2} \backslash\left\{\left|x_{i}\right| \leq \frac{1}{4},\left|x_{j}\right| \leq \frac{1}{4}\right\}\right)}\left|x_{j}\right|^{2} \rho(\mathbf{x}) \mathrm{d} \mathbf{x} \int_{\mathbb{R}^{m}} g_{\mathbf{r}}(\mathbf{w}, \tau)|\mathbf{w}|^{2} \mathrm{~d} \mathbf{w} \\
= & : J_{6,2,1}^{i, j}+J_{6,2,2}^{i, j} .
\end{aligned}
$$

By the second inequality in (2.23), the estimate for $J_{6,2,1}^{i, j}$ can be obtained by the same argument as that in (4.14):

$$
J_{6,2,1}^{i, j} \leq c(m)\|\varphi\|_{3} N^{3}|\mathbf{r}|^{2} t+c(m)\|\varphi\|_{3}\left(|\mathbf{r}|^{3} N^{\frac{2}{3}} \sigma_{j}^{\frac{2}{3}}+|\mathbf{r}| \sigma_{j}^{2}\right) \int_{0}^{t} \mathrm{~d} \tau \int_{\mathbb{R}^{m}}|\mathbf{w}|^{2} g_{\mathbf{r}}(\mathbf{w}, \tau) \mathrm{d} \mathbf{w}
$$

which gives $J_{6,2,1}^{i, j} \rightarrow 0\left(|\mathbf{r}| \rightarrow 0^{+}\right)$. By the assumptions of $\sigma_{i}^{2}=\lambda_{i} r_{i}$ and (2.5), we get

$$
\begin{aligned}
& \frac{c(m)\|\varphi\|_{3}}{|\mathbf{r}|} \int_{\mathbb{R}^{m-2} \times\left(\mathbb{R}^{2} \backslash\left\{\left|x_{i}\right| \leq \frac{1}{4},\left|x_{j}\right| \leq \frac{1}{4}\right\}\right)}\left|x_{j}\right|^{2} \frac{1}{\prod_{k=1}^{m} \sigma_{k}} \rho_{0}\left(\frac{x_{1}}{\sigma_{1}}, \cdots, \frac{x_{m}}{\sigma_{m}}\right) \mathrm{d} \mathbf{x} \\
\leq & c(m)\|\varphi\|_{3} \lambda_{j} \int_{\mathbb{R}^{m-2} \times\left(\mathbb{R}^{2} \backslash\left\{\left|y_{i}\right| \leq \frac{1}{\sqrt[4]{\lambda_{i} r_{i}}},\left|y_{j}\right| \leq \frac{1}{4 \sqrt{\lambda_{j} r_{j}}}\right\}\right)} y_{j}^{2} \rho_{0}(\mathbf{y}) \mathrm{d} \mathbf{y} .
\end{aligned}
$$

Note that $\int_{\mathbb{R}^{m}} y_{j}^{2} \rho_{0}(\mathbf{y}) \mathrm{d} \mathbf{y}=1$. Thus, $J_{6,2,2}^{i, j} \rightarrow 0$. This together with (4.18) and (4.20) shows that $J_{6} \rightarrow 0$ as $|\mathbf{r}| \rightarrow 0^{+}$.

(iii) The estimate for $J_{7}$. Let

$$
\begin{aligned}
J_{7}^{i, j}= & \frac{c(m)\|\varphi\|_{3}}{|\mathbf{r}|} \int_{0}^{t} \mathrm{~d} \tau \int_{\mathbb{R}^{m} \times \mathbb{R}^{m}} \rho(\mathbf{x}) \rho\left(\mathbf{x}_{*}\right) \mathrm{d} \mathbf{x} \mathrm{d} \mathbf{x}_{*} \int_{\mathbb{R}_{+}^{m}}\left(\prod_{k=1}^{m} \mathbb{1}_{\left\{w_{k *} \geq 0\right\}}\right) \\
& \times g_{\mathbf{r}}\left(\mathbf{w}_{*}, \tau\right) \mathrm{d} \mathbf{w}_{*} \int_{\bigcup_{\mu \in \Gamma} Q_{\mu}^{\left(\mathbf{x}, \mathbf{w}_{*}\right)}}\left(\left|x_{i}\right|^{3}\left|w_{i}\right|^{3} \wedge\left|x_{j}\right|^{2}\left|w_{j}\right|^{2}\right) g_{\mathbf{r}}(\mathbf{w}, \tau) \mathrm{d} \mathbf{w} .
\end{aligned}
$$

To prove $J_{7} \rightarrow 0$, we only need to prove that $J_{7}^{i, j} \rightarrow 0$ as $|\mathbf{r}| \rightarrow 0^{+}$. Similarly, we split $J_{7}^{i, j}$ into $J_{7,1}^{i, j}+J_{7,2}^{i, j}$,

$$
\begin{aligned}
J_{7,1}^{i, j}= & \frac{c(m)\|\varphi\|_{3}}{|\mathbf{r}|} \int_{0}^{t} \mathrm{~d} \tau \int_{\mathbb{R}^{m} \times \mathbb{R}^{m}} \rho(\mathbf{x}) \rho\left(\mathbf{x}_{*}\right) \mathrm{d} \mathbf{x} \mathrm{d} \mathbf{x}_{*} \int_{\mathbb{R}_{+}^{m}}\left(\prod_{k=1}^{m} \mathbb{1}_{\left\{w_{k *} \geq 0\right\}}\right) \\
& \times g_{\mathbf{r}}\left(\mathbf{w}_{*}, \tau\right) \mathrm{d} \mathbf{w}_{*} \int_{\bigcup_{\mu \in \Gamma_{i j}} Q_{\mu}^{\left(\mathbf{x}, \mathbf{w}_{*}\right)}}\left(\left|x_{i}\right|^{3}\left|w_{i}\right|^{3} \wedge\left|x_{j}\right|^{2}\left|w_{j}\right|^{2}\right) g_{\mathbf{r}}(\mathbf{w}, \tau) \mathrm{d} \mathbf{w}
\end{aligned}
$$

and

$$
\begin{aligned}
J_{7,2}^{i, j}= & \frac{c(m)\|\varphi\|_{3}}{|\mathbf{r}|} \int_{0}^{t} \mathrm{~d} \tau \int_{\mathbb{R}^{m} \times \mathbb{R}^{m}} \rho(\mathbf{x}) \rho\left(\mathbf{x}_{*}\right) \mathrm{d} \mathbf{x} \mathrm{d} \mathbf{x}_{*} \int_{\mathbb{R}_{+}^{m}}\left(\prod_{k=1}^{m} \mathbb{1}_{\left\{w_{k *} \geq 0\right\}}\right) \\
& \times g_{\mathbf{r}}\left(\mathbf{w}_{*}, \tau\right) \mathrm{d} \mathbf{w}_{*} \int_{\bigcup_{\mu \in\left(\Gamma \backslash \Gamma_{i j}\right)} Q_{\mu}^{\left(\mathbf{x}, \mathbf{w}_{*}\right)}}\left(\left|x_{i}\right|^{3}\left|w_{i}\right|^{3} \wedge\left|x_{j}\right|^{2}\left|w_{j}\right|^{2}\right) g_{\mathbf{r}}(\mathbf{w}, \tau) \mathrm{d} \mathbf{w} .
\end{aligned}
$$


Taking $\sigma_{i}^{2}=\lambda_{i} r_{i}$, by $(2.5)$ we have

$$
J_{7,1}^{i, j} \leq c(m)\|\varphi\|_{3} \int_{0}^{t} \mathrm{~d} \tau \int_{\mathbb{R}^{m}}\left(\sqrt{|\mathbf{r}|} \lambda_{i}^{\frac{3}{2}}\left|y_{i}\right|^{3} N^{3} \wedge \lambda_{j}\left|y_{j}\right|^{2} N^{2}\right) \rho_{0}(\mathbf{y}) \mathrm{d} \mathbf{y} .
$$

Recalling $a \wedge b=\min \{a, b\}$, one easily sees that

$$
\left(\sqrt{|\mathbf{r}|} \lambda_{i}^{\frac{3}{2}}\left|y_{i}\right|^{3} N^{3} \wedge \lambda_{j}\left|y_{j}\right|^{2} N^{2}\right) \rho_{0}(\mathbf{y}) \leq \lambda_{j} N^{2}\left|y_{j}\right|^{2} \rho_{0}(\mathbf{y}) .
$$

Since $\int_{\mathbb{R}^{m}} y_{j}^{2} \rho_{0}(\mathbf{y}) \mathrm{d} \mathbf{y}=1$ and $\lambda_{i}^{\frac{3}{2}} N^{3} \sqrt{|\mathbf{r}|} \rho_{0}(\mathbf{y})\left|y_{i}\right|^{3} \rightarrow 0$ as $|\mathbf{r}| \rightarrow 0^{+}$, it follows from the Lebesgue dominated convergence theorem [19] that $J_{7,1}^{i, j} \rightarrow 0$ as $|\mathbf{r}| \rightarrow 0^{+}$. It remains to show that $J_{7,2}^{i, j} \rightarrow 0$ as $|\mathbf{r}| \rightarrow 0^{+}$. Using the method as in (4.19), we get

$$
\begin{aligned}
& J_{7,2}^{i, j} \leq \frac{c(m)\|\varphi\|_{3}}{|\mathbf{r}|}\left(\int_{0}^{t} \mathrm{~d} \tau \int_{\mathbb{R}^{m-2} \times\left\{x_{i}, x_{j} \in \mathbb{R}^{1}|| x_{i}\left|\leq \frac{1}{4},\right| x_{j} \mid \leq \frac{1}{4}\right\}} \rho(\mathbf{x}) \mathrm{d} \mathbf{x} \int_{\mathbb{R}^{m}} g_{\mathbf{r}}\left(\mathbf{w}_{*}, \tau\right)\right. \\
& \times\left[8\left|x_{i}\right|^{3}\left(4^{3} N^{3}+4^{3} r_{i}^{3}\left|w_{i *}\right|^{3}\right) \wedge 4\left|x_{j}\right|^{2}\left(4^{2} N^{2}+4^{2} r_{j}^{2}\left|w_{j *}\right|^{2}\right)\right] \mathrm{d} \mathbf{w}_{*} \\
& \left.+\int_{0}^{t} \mathrm{~d} \tau \int_{\mathbb{R}^{m-2} \times\left(\mathbb{R}^{2} \backslash\left\{x_{i}, x_{j} \in \mathbb{R}^{1}|| x_{i}\left|\leq \frac{1}{4},\right| x_{j} \mid \leq \frac{1}{4}\right\}\right)}\left|x_{j}\right|^{2} \rho(\mathbf{x}) \mathrm{d} \mathbf{x} \int_{\mathbb{R}^{m}}|\mathbf{w}|^{2} g_{\mathbf{r}}(\mathbf{w}, \tau) \mathrm{d} \mathbf{w}\right) .
\end{aligned}
$$

By the same argument as that for the estimates for $J_{5}^{i, j}, J_{6,2,2}^{i, j}, J_{7,1}^{i, j}$, we get $J_{7,2}^{i, j} \rightarrow 0$ as $|\mathbf{r}| \rightarrow 0^{+}$.

Therefore we obtain that $J_{7} \rightarrow 0$ as $|\mathbf{r}| \rightarrow 0^{+}$by the estimates of $J_{7,1}^{i, j}$ and $J_{7,2}^{i, j}$. Summing up all the above estimates in (i)-(iii) and combining (4.11)-(4.12), we conclude that $\mathcal{R}_{1}(\mathbf{r}) \rightarrow 0$ as $|\mathbf{r}| \rightarrow 0^{+}$.

Step 2: Proof of $\mathcal{R}_{2}(\mathbf{r}) \rightarrow 0$ as $|\mathbf{r}| \rightarrow 0^{+}$. By (2.23), (2.28), (4.6), and (4.9)(4.10), we have

$$
\begin{aligned}
\left|\mathcal{R}_{2}(\mathbf{r})\right| & \leq \frac{2\|\varphi\|_{3}}{|\mathbf{r}|} \int_{0}^{t} \mathrm{~d} \tau \int_{\mathbb{R}^{m} \times \mathbb{R}^{m}}\left[\sum_{j=1}^{m} \mathbb{1}_{\left\{x_{j}<r_{j}-1\right\}}+\sum_{j=1}^{m} \mathbb{1}_{\left\{x_{j *}<r_{j}-1\right\}}\right] \rho(\mathbf{x}) \\
& \times \rho\left(\mathbf{x}_{*}\right) \mathrm{d} \mathbf{x} \mathrm{d} \mathbf{x}_{*} \int_{\mathbb{R}_{+}^{m}} g_{\mathbf{r}}\left(\mathbf{w}_{*}, \tau\right) \mathrm{d} \mathbf{w}_{*} \int_{\bigcup_{\mu \in \Gamma} Q_{\mu}^{\left(\mathbf{x}, \mathbf{w}_{*}\right)}}\left[\left(\sum_{i=1}^{m}\left|w_{i}^{\prime}-w_{i}\right|\right) \wedge 1\right] g_{\mathbf{r}}(\mathbf{w}, \tau) \mathrm{d} \mathbf{w} .
\end{aligned}
$$

For any fixed $i, j$, we get

$$
\begin{aligned}
& \frac{1}{|\mathbf{r}|} \int_{0}^{t} \mathrm{~d} \tau \int_{\mathbb{R}^{m} \times \mathbb{R}^{m}} \mathbb{1}_{\left\{x_{j}<r_{j}-1\right\}} \rho(\mathbf{x}) \\
& \quad \times \rho\left(\mathbf{x}_{*}\right) \mathrm{d} \mathbf{x} \mathrm{d} \mathbf{x}_{*} \int_{\mathbb{R}_{+}^{m}} g_{\mathbf{r}}\left(\mathbf{w}_{*}, \tau\right) \mathrm{d} \mathbf{w}_{*} \int_{\bigcup_{\mu \in \Gamma} Q_{\mu}^{\left(\mathbf{x}, \mathbf{w}_{*}\right)}} g_{\mathbf{r}}(\mathbf{w}, \tau)\left(\left|x_{i}\right|\left|w_{i}\right| \wedge 1\right) \mathrm{d} \mathbf{w} \\
& \leq \frac{1}{|\mathbf{r}|\left(1-r_{j}\right)^{2}} \int_{0}^{t} \mathrm{~d} \tau \int_{\mathbb{R}^{m}}\left(\left|x_{i}\right|\left|x_{j}\right|^{2} N \wedge\left|x_{j}\right|^{2}\right) \rho(\mathbf{x}) \mathrm{d} \mathbf{x} \\
& +\frac{1}{|\mathbf{r}|\left(1-r_{j}\right)^{2}} \int_{0}^{t} \int_{\mathbb{R}^{m-1} \times\left\{x_{i} \in \mathbb{R}^{1}|| x_{i} \mid \leq \frac{1}{4}\right\}} \rho(\mathbf{x}) \mathrm{d} \mathbf{x} \int_{\mathbb{R}^{m}} g_{\mathbf{r}}\left(\mathbf{w}_{*}, \tau\right) \\
& \quad+\frac{1}{|\mathbf{r}|\left(1-r_{j}\right)^{2}} \int_{0}^{t} \mathrm{~d} \tau \int_{\mathbb{R}^{m-1} \times\left\{x_{i} \in \mathbb{R}^{1}|| x_{i} \mid>\frac{1}{4}\right\}}\left|x_{j}\right|^{2} \rho(\mathbf{x}) \mathrm{d} \mathbf{x} .
\end{aligned}
$$


The same argument as that for the previous estimates in (4.21) and (4.25) shows that all the integrals on the right-hand side of (4.28) converge to zero as $|\mathbf{r}| \rightarrow 0^{+}$. Moreover, by (2.5) we have

$$
\begin{aligned}
& \frac{1}{|\mathbf{r}|} \int_{0}^{t} \mathrm{~d} \tau \int_{\mathbb{R}^{m} \times \mathbb{R}^{m}} \mathbb{1}_{\left\{x_{j *}<r_{j}-1\right\}} \rho(\mathbf{x}) \\
& \quad \times \rho\left(\mathbf{x}_{*}\right) \mathrm{d} \mathbf{x} \mathrm{d} \mathbf{x}_{*} \int_{\mathbb{R}_{+}^{m}} g_{\mathbf{r}}\left(\mathbf{w}_{*}, \tau\right) \mathrm{d} \mathbf{w}_{*} \int_{\bigcup_{\mu \in \Gamma} Q_{\mu}^{\left(\mathbf{x}, \mathbf{w}_{*}\right)}} g_{\mathbf{r}}(\mathbf{w}, \tau)\left(\left|x_{i}\right|\left|w_{i}\right| \wedge 1\right) \mathrm{d} \mathbf{w} \\
& \leq \frac{4 \sigma_{i} \sigma_{j}^{2}}{|\mathbf{r}|} \int_{0}^{t}\left(\int_{\mathbb{R}^{m}}|\mathbf{w}|^{2} g_{\mathbf{r}}(\mathbf{w}, \tau) \mathrm{d} \mathbf{w}\right)^{\frac{1}{2}} \mathrm{~d} \tau
\end{aligned}
$$

Finally grouping the estimates in Step 2, we conclude that $\mathcal{R}_{2}(\mathbf{r}) \rightarrow 0$ as $|\mathbf{r}| \rightarrow 0^{+}$under the assumption of $\sigma_{i}^{2}=\lambda_{i} r_{i}$.

Step 3: Proof of $L^{1}$-weak compactness of $\left\{g_{\mathbf{r}}(\cdot, t)\right\}_{\mathbf{r}, t}$. We only need to verify the Dunford-Pettis compactness criterion [11] for weak compactness in $L^{1}$. It follows by Lemma 3.3 and the Hölder inequality that

$$
\sup _{|\mathbf{r}| \leq \frac{1}{2}, t \in[0, T]} \int_{E} g_{\mathbf{r}}(\mathbf{w}, t) \mathrm{d} \mathbf{w} \rightarrow 0 \quad \text { as } \quad m(E) \rightarrow 0 \quad(\forall 0<T<\infty) .
$$

And the inequality (3.1) implies that

$$
\sup _{0<|\mathbf{r}| \leq \frac{1}{2}, t \in[0, T]} \int_{|\mathbf{w}|>R} g_{\mathbf{r}}(\mathbf{w}, t) \mathrm{d} \mathbf{w} \rightarrow 0 \quad(R \rightarrow+\infty) .
$$

Moreover, using the fact that $\int_{\mathbb{R}^{m}} g_{\mathbf{r}}(\mathbf{w}, t) \mathrm{d} \mathbf{w}=\int_{\mathbb{R}^{m}} f(\mathbf{w}, 0) \mathrm{d} \mathbf{w}$, it follows that $\left\{g_{\mathbf{r}}(\mathbf{w}, t)\right\}_{\mathbf{r}, t}$ is $L^{1}$-weakly compact.

Just as in [16], let

$$
\Lambda_{n}[\varphi](t)=\int_{\mathbb{R}^{m}} \varphi(\mathbf{w}) g_{\mathbf{r}_{n}}(\mathbf{w}, t) \mathrm{d} \mathbf{w}, \quad \varphi \in C_{c}^{\infty}\left(\mathbb{R}_{>0}^{m}\right) .
$$

Recall that $|\mathbf{r}|=\max _{1 \leq i \leq 1}\left\{r_{i}\right\}$. For any sequence $\left\{\left|\mathbf{r}_{n}\right|\right\} \rightarrow 0(n \rightarrow \infty)$, applying the diagonal procedure we obtain a subsequence, still denoted by $\{n\}$, such that $\Lambda[\varphi](\bar{t}):=$ $\lim _{n \rightarrow \infty} \Lambda_{n}[\varphi](\bar{t})$ exists for all $\varphi \in C_{c}^{\infty}\left(\mathbb{R}_{>0}^{m}\right)$ and all $\bar{t} \in \mathbf{Q}_{+}$. Here $\mathbf{Q}_{+}$is the set of nonnegative rational numbers. So it remains to prove that this limit also exists for all $t \in[0, \infty)$. Actually, we only need to prove the following

$$
\sup _{\left|\mathbf{r}_{n}\right|<\frac{1}{2}}\left|\Lambda_{n}[\varphi]\left(t_{1}\right)-\Lambda_{n}[\varphi]\left(t_{2}\right)\right| \rightarrow 0 \quad \text { as } \quad\left|t_{1}-t_{2}\right| \rightarrow 0 .
$$

Under the assumptions (2.28), (4.2)-(4.3), taking $\sigma_{i}^{2}=\lambda_{i} r_{i}$ we have

$$
\begin{aligned}
& \quad\left|\int_{\mathbb{R}^{m}} \varphi(\mathbf{w}) g_{\mathbf{r}}\left(\mathbf{w}, t_{1}\right) d \mathbf{w}-\int_{\mathbb{R}^{m}} \varphi(\mathbf{w}) g_{\mathbf{r}}\left(\mathbf{w}, t_{2}\right) \mathrm{d} \mathbf{w}\right| \\
& \leq \sum_{i=1}^{m} 8 \lambda_{i}\|\varphi\|_{3}\left|t_{2}-t_{1}\right|+2\|\varphi\|_{3}\left|\int_{t_{1}}^{t_{2}}\left(\int_{\mathbb{R}^{m}}|\mathbf{w}|^{2} g_{\mathbf{r}}(\mathbf{w}, \tau) \mathrm{d} \mathbf{w}\right)^{\frac{1}{2}} \mathrm{~d} \tau\right| \\
& \quad+\left.c(m)\|\varphi\|_{3}\left(1+\sum_{i=1}^{m} \lambda_{i}\right)\left|\int_{t_{1}}^{t_{2}} \int_{\mathbb{R}^{m}}\right| \mathbf{w}\right|^{2} g_{\mathbf{r}}(\mathbf{w}, \tau) \mathrm{d} \mathbf{w} \mathrm{d} \tau \mid \\
& \leq b e^{\frac{c}{\mathbf{r} \mid}\left(t_{1} \vee t_{2}\right)}\|\varphi\|_{3}\left|t_{1}-t_{2}\right|
\end{aligned}
$$


for every $\varphi \in C_{c}^{\infty}\left(\mathbb{R}_{>0}^{m}\right)$ and arbitrary $t_{1}, t_{2}$. Here $t_{1} \vee t_{2}=\max \left\{t_{1}, t_{2}\right\}$ and $b$ is a constant depending only on $\lambda_{i}, m$, and $\int_{\mathbb{R}^{m}}|\mathbf{w}|^{2} g(\mathbf{w}, 0) \mathrm{d} \mathbf{w}$. In addition, it is shown that $c /|\mathbf{r}|$ is bounded by a constant independent of $\mathbf{r}$ under the assumption of $\sigma_{i}^{2}=\lambda_{i} r_{i}$ by the formula (2.33). Then (4.33) follows from (4.34). Therefore, we have $\Lambda[\varphi](t):=\lim _{\bar{t} \rightarrow t} \Lambda[\varphi](\bar{t})$ for all $\varphi \in C_{c}^{\infty}\left(\mathbb{R}_{>0}^{m}\right)$ and all $t \in[0, \infty)$. Moreover, since $C_{c}^{\infty}\left(\mathbb{R}_{>0}^{m}\right)$ is dense in $C_{c}\left(\mathbb{R}_{+}^{m}\right)$, it follows that $\lim _{n \rightarrow \infty} \Lambda_{n}[\psi](t)=\Lambda[\psi](t)$ holds for all $\psi \in C_{c}\left(\mathbb{R}_{+}^{m}\right)$ and all $t \in[0, \infty)$. Then the Riesz representation theorem [22] and $L^{1}$ weak compactness of $\left\{g_{\mathbf{r}}(\mathbf{w}, t)\right\}_{\mathbf{r}, t}$ ensure that there exists $g \in L^{1}\left(\mathbb{R}_{+}^{m}\right)$ such that $\Lambda[\psi](t)=\int_{\mathbb{R}^{m}} \psi(\mathbf{w}) g(\mathbf{w}, t)$ d $\mathbf{w}$, i.e.

$$
\lim _{n \rightarrow \infty} \int_{\mathbb{R}^{m}} \psi(\mathbf{w}) g_{\mathbf{r}_{n}}(\mathbf{w}, t) \mathrm{d} \mathbf{w}=\int_{\mathbb{R}^{m}} \psi(\mathbf{w}) g(\mathbf{w}, t) \mathrm{d} \mathbf{w}, \quad \forall \psi \in C_{c}\left(\mathbb{R}_{+}^{m}\right), \quad \forall t \in[0,+\infty) .
$$

Combining the conclusions of $\mathcal{R}_{1}(\mathbf{r}), \mathcal{R}_{2}(\mathbf{r})$ with Lemma 3.2, and taking now the weak limit in (4.2) as $n \rightarrow \infty$, we find

$$
\begin{aligned}
\int_{\mathbb{R}^{m}} \varphi(\mathbf{w}) g(\mathbf{w}, t) \mathrm{d} \mathbf{w}= & \int_{\mathbb{R}^{m}} \varphi(\mathbf{w}) g(\mathbf{w}, 0) \mathrm{d} \mathbf{w}+\int_{0}^{t} \int_{\mathbb{R}^{m}} g(\mathbf{w}, \tau)\left[\sum_{i=1}^{m} q_{i}\left(S_{i}-w_{i}\right) \frac{\partial \varphi(\mathbf{w})}{\partial w_{i}}\right. \\
& \left.+\frac{1}{2} \sum_{i=1}^{m} \sum_{j=1}^{m} \kappa_{i j} \sqrt{q_{i} q_{j} \lambda_{i} \lambda_{j}} w_{i} w_{j} \frac{\partial^{2} \varphi(\mathbf{w})}{\partial w_{i} \partial w_{j}}\right] \mathrm{d} \mathbf{w} \mathrm{d} \tau
\end{aligned}
$$

for all $t \geq 0$ and all $\varphi \in C_{c}^{\infty}\left(\mathbb{R}_{>0}^{m}\right)$. Here $S_{i}=\int_{\mathbb{R}^{m}} w_{i} g(\mathbf{w}, 0) \mathrm{d} \mathbf{w}, q_{i}>0$ is given in (3.9) and $\kappa_{i j}$ is the correlation coefficient between the rate of return for asset $i$ and asset $j$. Taking the derivative with respect to $t$ in (4.35), we obtain

$$
\begin{aligned}
\frac{d}{d t} \int_{\mathbb{R}^{m}} \varphi(\mathbf{w}) g(\mathbf{w}, t) \mathrm{d} \mathbf{w}= & \int_{\mathbb{R}^{m}} g(\mathbf{w}, t)\left[\sum_{i=1}^{m} q_{i}\left(S_{i}-w_{i}\right) \frac{\partial \varphi(\mathbf{w})}{\partial w_{i}}\right. \\
& \left.+\frac{1}{2} \sum_{i=1}^{m} \sum_{j=1}^{m} \kappa_{i j} \sqrt{q_{i} q_{j} \lambda_{i} \lambda_{j}} w_{i} w_{j} \frac{\partial^{2} \varphi(\mathbf{w})}{\partial w_{i} \partial w_{j}}\right] \mathrm{d} \mathbf{w} .
\end{aligned}
$$

Moreover, it is easily seen that

$$
\begin{aligned}
& \frac{d}{d t} \int_{\mathbb{R}^{m}} \varphi(\mathbf{w}, t) g(\mathbf{w}, t) \mathrm{d} \mathbf{w} \\
= & \int_{\mathbb{R}^{m}}\left(\frac{\partial \varphi}{\partial t}(\mathbf{w}, t)\right) g(\mathbf{w}, t) \mathrm{d} \mathbf{w} \\
& +\int_{\mathbb{R}^{m}} g(\mathbf{w}, t)\left[\sum_{i=1}^{m} q_{i}\left(S_{i}-w_{i}\right) \frac{\partial \varphi(\mathbf{w}, t)}{\partial w_{i}}\right. \\
& \left.+\frac{1}{2} \sum_{i=1}^{m} \sum_{j=1}^{m} \kappa_{i j} \sqrt{q_{i} q_{j} \lambda_{i} \lambda_{j}} w_{i} w_{j} \frac{\partial^{2} \varphi(\mathbf{w}, t)}{\partial w_{i} \partial w_{j}}\right] \mathrm{d} \mathbf{w}, \\
& \forall \varphi \in C_{c}^{\infty}\left(\mathbb{R}_{>0}^{m} \times[0, \infty)\right) .
\end{aligned}
$$


In fact, for any $t_{0} \in[0, \infty)$, we have for $t \geq 0$ with $t \neq t_{0}$ that

$$
\begin{aligned}
& \frac{1}{t-t_{0}}\left(\int_{\mathbb{R}^{m}} \varphi(\mathbf{w}, t) g(\mathbf{w}, t) \mathrm{d} \mathbf{w}-\int_{\mathbb{R}^{m}} \varphi\left(\mathbf{w}, t_{0}\right) g\left(\mathbf{w}, t_{0}\right) \mathrm{d} \mathbf{w}\right) \\
= & \frac{1}{t-t_{0}} \int_{\mathbb{R}^{m}}\left(\int_{t_{0}}^{t} \frac{\partial}{\partial \tau} \varphi(\mathbf{w}, \tau) \mathrm{d} \tau\right) g(\mathbf{w}, t) \mathrm{d} \mathbf{w}+\frac{1}{t-t_{0}} \int_{t_{0}}^{t} \int_{\mathbb{R}^{m}} g(\mathbf{w}, \tau) \\
& {\left[\sum_{i=1}^{m} q_{i}\left(S_{i}-w_{i}\right) \frac{\partial \varphi\left(\mathbf{w}, t_{0}\right)}{\partial w_{i}}+\frac{1}{2} \sum_{i=1}^{m} \sum_{j=1}^{m} \kappa_{i j} \sqrt{q_{i} q_{j} \lambda_{i} \lambda_{j}} w_{i} w_{j} \frac{\partial^{2} \varphi\left(\mathbf{w}, t_{0}\right)}{\partial w_{i} \partial w_{j}}\right] \mathrm{d} \mathbf{w} \mathrm{d} \tau } \\
\rightarrow & \int_{\mathbb{R}^{m}}\left(\frac{\partial \varphi}{\partial t}\left(\mathbf{w}, t_{0}\right)\right) g\left(\mathbf{w}, t_{0}\right) \mathrm{d} \mathbf{w}+\int_{\mathbb{R}^{m}} g\left(\mathbf{w}, t_{0}\right)\left[\sum_{i=1}^{m} q_{i}\left(S_{i}-w_{i}\right) \frac{\partial \varphi\left(\mathbf{w}, t_{0}\right)}{\partial w_{i}}\right. \\
& \left.+\frac{1}{2} \sum_{i=1}^{m} \sum_{j=1}^{m} \kappa_{i j} \sqrt{q_{i} q_{j} \lambda_{i} \lambda_{j}} w_{i} w_{j} \frac{\partial^{2} \varphi\left(\mathbf{w}, t_{0}\right)}{\partial w_{i} \partial w_{j}}\right] \mathrm{d} \mathbf{w} \quad\left(t \rightarrow t_{0}\right) .
\end{aligned}
$$

This gives the weak form of the Fokker-Planck equation

$$
\frac{\partial g(\mathbf{w}, t)}{\partial t}=\sum_{i=1}^{m} q_{i} \frac{\partial}{\partial w_{i}}\left[\left(w_{i}-S_{i}\right) g(\mathbf{w}, t)\right]+\frac{1}{2} \sum_{i=1}^{m} \sum_{j=1}^{m} \kappa_{i j} \sqrt{q_{i} q_{j} \lambda_{i} \lambda_{j}} \frac{\partial^{2}}{\partial w_{i} \partial w_{j}}\left[w_{i} w_{j} g(\mathbf{w}, t)\right]
$$

with the initial datum $g(\mathbf{w}, 0)$.

REMARK 4.4. The uniqueness of weak solutions $g(\mathbf{w}, t)$ of the Fokker-Planck Equation (4.1) is unknown. The main reason is that the complicated structure of the kernels of the model, such as characteristic functions, forces us to use the smooth test function space $C_{c}^{\infty}\left(\mathbb{R}_{>0}^{m}\right)$ rather than $L^{2}\left(\mathbb{R}^{m}\right)$. Additionally, from our proof of Lemma 3.3 one can see that it is very hard to replace the $L^{2}$ condition with the $L^{1}$ condition when proving the $L^{1}$-weak compactness of $\left\{g_{\mathbf{r}}(\mathbf{w}, t)\right\}_{\mathbf{r}, t}$ for the same reason.

\section{Conclusion}

From J.Mossin [18], we know that portfolios can be denoted by vectors. Following the ideas in [9], we set up a multi-dimensional model by the theories and methods in [3]. The model might be helpful to describe the time evolution of the portfolio distribution in a financial market. The main result, Theorem 4.1, which is obtained in a suitable way, demonstrates that the mild solution is approximated by a weak solution of the multi-dimensional Fokker-Planck equation at large times.

Moreover, further research about the portfolio distribution might give us guidance in investments and other related fields.

Acknowledgement. I am grateful to Professor Xuguang Lu for his encouragement and helpful discussions.

\section{REFERENCES}

[1] L. Arkeryd, On the Boltzmann equation. Part I: Existence, Arch. Rat. Mech. Anal, 45, 1-16, 1972.

[2] M. Bisi, G. Spiga, and G. Toscani, Kinetic models of conservative economies with wealth redistribution, Commun. Math. Sci., 7, 901-916, 2009.

[3] C. Cercignani, R. Illner, and M. Pulvirenti, The Mathematical Theory of Dilute Gases, Springer, 1994.

[4] A. Chakraborti, Distributions of money in model markets of economy, Int. J. Mod. Phys. C, 13, 1315-1321, 2002. 
[5] A. Chakraborti and B. Chakrabarti, Statistical mechanics of money: How saving propensity affects its distribution, Eur. Phys. J. B., 17, 167-170, 2000.

[6] A. Chatterjee and B. Chakrabarti, Kinetic exchange models for income and wealth distributions, Eur. Phys. J. B, 60, 135-149, 2007.

[7] A. Chatterjee, B. K. Chakrabarti, and S. S. Manna, Pareto law in a kinetic model of market with random saving propensity, Physica A: Stat. Mech. Appl., 335, 155-163, 2004.

[8] S. Cordier, L. Pareschi, and C. Piatecki, Mesoscopic modelling of financial markets, J. Stat. Phys., 134, 161-184, 2009.

[9] S. Cordier, L. Pareschi, and G. Toscani, On a kinetic model for a simple market economy, J. Stat. Phys., 120, 253-277, 2005.

[10] A. Drăgulescu and V. Yakovenko, Statistical mechanics of money, Eur. Phys. J. B., 17, 723-729, 2000.

[11] N. Dunford and J. Schwartz, Linear Operators: General Theory, Interscience publishers, 1958.

[12] B. Düring, D. Matthes, and G. Toscani, Kinetic equations modelling wealth redistribution: A comparison of approaches, Phys. Rev. E, 78, 056103, 2008.

[13] B. Düring and G. Toscani, Hydrodynamics from kinetic models of conservative economies, Physica A: Stat. Mech. Appl., 384, 493-506, 2007.

[14] S. Ispolatov, P. Krapivsky, and S. Redner, Wealth distributions in asset exchange models, Eur. Phys. J. B., 2, 267-276, 1998.

[15] X. Lu, A modified Boltzmann equation for Bose-Einstein particles: Isotropic solutions and long-time behavior, J. Stat. Phys., 98, 1335-1394, 2000.

[16] X. Lu, On isotropic distributional solutions to the Boltzmann equation for Bose-Einstein particles, J. Stat. Phys., 116, 1597-1649, 2004.

[17] D. Matthes and G. Toscani, On steady distributions of kinetic models of conservative economies, J. Stat. Phys., 130, 1087-1117, 2008.

[18] J. Mossin, Equilibrium in a capital asset market, Econometrica: Journal of the Econometric Society, 768-783, 1966.

[19] A. Mukherjea and K. Pothoven, Real and functional analysis, Plenum Press, 1978.

[20] L. Pareschi and G. Toscani, Self-similarity and power-like tails in nonconservative kinetic models, J. Stat. Phys., 124, 747-779, 2006.

[21] V. Pareto, Cours d'Economie Politique, Librairie Droz, 1964.

[22] W. Rudin, Real and Complex Analysis, Tata McGraw-Hill, 2006.

[23] G. Toscani, One-dimensional kinetic models of granular flows, RAIRO Modél Math. Anal. Numér., 34, 1277-1291, 2000.

[24] R. Alexandre and C. Villani, On the Landau approximation in plasma physics, in Annales de l'Institut Henri Poincare (C) Non Linear Analysis, vol. 21, Elsevier, 61-95, 2004.

[25] V. Yakovenko and J. Rosser Jr, Colloquium: Statistical mechanics of money, wealth, and income, Rev. Mod. Phys., 81, 1703, 2009. 